Linköping Studies in Science and Technology. Dissertations.

No. 1699

\title{
A class of infinite dimensional stochastic processes with unbounded diffusion and its associated Dirichlet forms
}

\author{
John Karlsson
}

Department of Mathematics

Linköping University, SE-581 83 Linköping, Sweden Linköping 2015 
Linköping Studies in Science and Technology. Dissertations.

No. 1699

A class of infinite dimensional stochastic processes with unbounded diffusion and its associated Dirichlet forms

John Karlsson

$$
\begin{gathered}
\text { john. karlsson@liu.se } \\
\text { www.mai.liu.se } \\
\text { Mathematical Statistics } \\
\text { Department of Mathematics } \\
\text { Linköping University } \\
\text { SE-58183 Linköping } \\
\text { Sweden }
\end{gathered}
$$

ISBN 978-91-7685-966-7

ISSN 0345-7524

Copyright (C) 2015 John Karlsson, unless otherwise noted

Printed by LiU-Tryck, Linköping, Sweden 2015 




\section{Abstract}

This thesis consists of two papers which focuses on a particular diffusion type Dirichlet form

$$
\mathcal{E}(F, G)=\int\langle A D F, D G\rangle_{\mathbb{H}} d \nu
$$

where $A=\sum_{i=1}^{\infty} \lambda_{i}\left\langle S_{i}, \cdot\right\rangle_{\mathbb{H}} S_{i}$. Here $S_{i}, i \in \mathbb{N}$, is the basis in the Cameron-Martin space, $\mathbb{H}$, consisting of the Schauder functions, and $\nu$ denotes the Wiener measure.

In Paper I, we let $\lambda_{i}, i \in \mathbb{N}$, vary over the space of wiener trajectories in a way that the diffusion operator $A$ is almost everywhere an unbounded operator on the CameronMartin space. In addition we put a weight function $\varphi$ on the Wiener measure $\nu$ and show that under these changes of the reference measure, the Malliavin derivative and divergence are closable operators with certain closable inverses. It is then shown that under certain conditions on $\lambda_{i}, i \in N$, and these changes of reference measure, the Dirichlet form is quasi-regular. This is done first in the classical Wiener space and then the results are transferred to the Wiener space over a Riemannian manifold.

Paper II focuses on the case when $\lambda_{i}, i \in \mathbb{N}$, is a sequence of non-decreasing real numbers. The process $X$ associated to $(\mathcal{E}, D(\mathcal{E}))$ is then an infinite dimensional OrnsteinUhlenbeck process. In this case we show that the distributions of a sequence of certain finite dimensional Ornstein-Uhlenbeck processes converge weakly to the distribution of the infinite dimensional Ornstein-Uhlenbeck process. We also investigate the quadratic variation for this process, both in the classical sense and in the recent framework of stochastic calculus via regularization. Since the process is Banach space valued, the tensor quadratic variation is an appropriate tool to establish the Itô formula for the infinite dimensional Ornstein-Uhlenbeck process $X$. Sufficient conditions are presented for the scalar as well as the tensor quadratic variation to exist. 



\section{Populärvetenskaplig sammanfattning}

En stokastisk process är en matematisk representation av hur ett slumpmässigt system utvecklas under tid. Exempelvis är värdet på en aktie en endimensionell process och positionen på en partikel som rör sig slumpmässigt i rummet är en tredimensionell process. Det är svårare att föreställa sig och analysera processer som tar värden i oändligdimensionella rum men det finns olika sätt att behandla problemet matematiskt. Ett sätt är att studera så kallade Dirichletformer. En Dirichletform är ett matematiskt objekt inom området potentialteori. Genom att använda sig av en sådan framställning får man tillgång till de verktyg som finns inom potentialteorins ämnesområde vilket kan göra det matematiska arbetet enklare.

Det visar sig att endast vissa Dirichletformer har en motsvarande stokastisk process. I det första pappret i den här avhandlingen behandlas en viss typ av Dirichletformer där den så kallade diffusionen ökar för varje dimension. Man kan säga att diffusionen är hastigheten på slumprörelsen. Vi visar hur snabbt diffusionen får öka för att slutprocessen ska vara väldefinierad. Pappret behandlar även fallet då processen lever i ett krökt rum, på en mångfald, som exempel kan man tänka sig ytan av en ballong istället för ytan på ett papper.

I det andra pappret ligger fokus på den associerade oändligdimensionella processen som vi kallar $X$. Här visas bland annat att man under vissa omständigheter kan approximera $X$ med ändligdimensionella processer. Vi beräknar också processens kvadratiska variation som är ett mått på hur mycket processens värde fluktuerar under tid. Genom att visa att en speciell typ av kvadratisk variation existerar kan vi även presentera en Itô formel, ett hjälpsamt verktyg för att bedriva analys på processen. 



\section{Acknowledgments}

I would like to express my thanks to Linköping University and the department of mathematics for the opportunity I have had to work there. A special thanks goes to my main supervisor Jörg-Uwe Löbus and my co-supervisor Torkel Erhardsson for all the help and support I have received during this $\mathrm{PhD}$ project. Thanks also to the administrative personnel and co-workers at MAI that I have been fortunate to share this department with.

Thanks goes to my fellow PhD students that have been a source of inspiration and good times. A special mention goes to my friend Marcus Kardell that I have now known and had countless discussions with for almost five years.

I would also like to thank my family, other friends, and anyone else I might have forgot to mention, these pages would be full if I were to write down the names of everyone deserving a mention.

Finally, once again I would like to thank my main supervisor Jörg-Uwe Löbus for all the effort and time he put into this work, without him this could never have been done. 



\section{Contents}

Abstract ........................ . . .

Populärvetenskaplig sammanfattning . . . . . . . . . . . . . vii

Acknowledgements ................... ix

\section{Introduction}

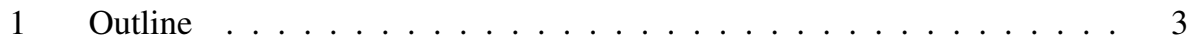

1.1 Motivation for the papers . . . . . . . . . . 3

$1.2 \quad$ Paper I specifics . . . . . . . . . . . . . . . . . 4

1.3 Paper II specifics . . . . . . . . . . . . . . . 5

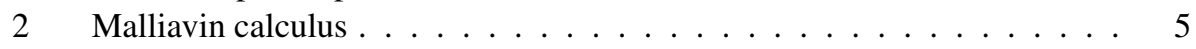

2.1 General framework . . . . . . . . . . . . . 5

2.2 Hermite polynomials . . . . . . . . . . . . . 6

2.3 Wiener Chaos . . . . . . . . . . . . . . 6

2.4 Multiple stochastic integrals .............. 7

2.5 Derivative operator . . . . . . . . . . . . 8

2.6 Divergence operator . . . . . . . . . . . . . 9

3 Dirichlet forms . . . . . . . . . . . . . . . . 10

4 Stochastic integration via regularization .............. . . . . . . . . . . . . . .

4.1 One dimensional case . . . . . . . . . . . . . . . . . 12

4.2 Infinite dimensional case . . . . . . . . . . . . . . . . . 13

5 The geometric Cameron-Martin formula . . . . . . . . . . . . . 15

$5.1 \quad$ Manifolds . . . . . . . . . . . . . . . . . 15

5.2 Riemannian connection . . . . . . . . . . . . . . . 18

5.3 The orthonormal bundle . . . . . . . . . . . . . 20

5.4 Brownian motion on a smooth Riemannian manifold . . . . . . . 21

5.5 Analysis on the path space . . . . . . . . . . . . 23

5.6 Directional derivative, gradient, divergence and integration by parts 27

5.7 Tensors and Ricci curvature . . . . . . . . . . . . . . . 29

References ........................ 31

\section{Paper I}

1 Introduction . . . . . . . . . . . . . . . . . . . 37

2 Definitions ........................ 40

3 Closability of derivative, divergence, and their inverses . . . . . . . . . 42

4 Closability of the bilinear form . . . . . . . . . . . . . . 50 


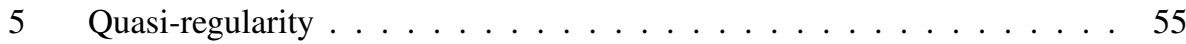

6 Transferring the results to a geometric setting . . . . . . . . . . 59

References . . . . . . . . . . . . . . . . . 67

\section{Paper II}

1 Introduction . . . . . . . . . . . . . . . . . . 73

$1.1 \quad$ Main results . . . . . . . . . . . . . . . . . 75

1.2 Some basic definitions . . . . . . . . . . . . . 77

2 Finite dimensional approximation of the infinite dimensional process . . . 78

3 Scalar quadratic variation . . . . . . . . . . . . . . . . . 85

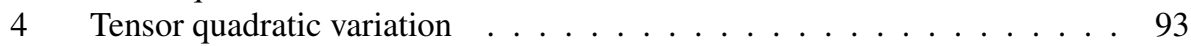

5 Itô's formula . . . . . . . . . . . . . . . . . . . . . . 99

A Appendix: Some lemmas in extreme value theory . . . . . . . . . 107

References . . . . . . . . . . . . . . . . . . 112 


\section{Introduction}





\section{Outline}

This thesis consists of two main parts. The first part is an introductory part containing key concepts of the theory used in the second part. The second part contains two papers, A class of infinite dimensional stochastic processes with unbounded diffusion and Infinite dimensional Ornstein-Uhlenbeck processes with unbounded diffusion - Approximation, quadratic variation, and Itô formula, written at Linköping university during the years 2012-2015. Both papers are joint work with Jörg-Uwe Löbus. What follows below is a short summary of the different topics in part one.

\section{Malliavin calculus}

The purpose of this section is to provide a brief introduction to some notions in Malliavin calculus. Concepts such as the Malliavin derivative are essential for the definition of the bilinear Dirichlet form which is the main object of this study. We also present the Wiener chaos decomposition as we make use of it in Paper I.

\section{Dirichlet forms}

This section is devoted to the notion of Dirichlet forms. The section contains the basic definitions needed for understanding the theorem of Ma/Röckner which provides the connection between Dirichlet forms and stochastic processes.

\section{Stochastic integration via regularization}

Here we present some main ideas of the recent theory of stochastic integration via regularization. These concepts are used in Paper II in order to obtain a corresponding Itô formula to the stochastic process being studied.

\section{Differential geometry}

This is a presentation of basic notions in differential geometry. These concepts are then used to define Brownian motion on a Riemannian manifold. This theory is used in Paper I in the final section which transfers results of the flat case to a geometric setting.

\subsection{Motivation for the papers}

The theory of Dirichlet forms is a subject showing that certain bilinear forms can serve as a connection between analysis and probability. This connection to probability was established by the fundamental work of Silverstein and Fukushima during the 1970s (see [Meyer, 2009]). In particular, Fukushima showed that if a Dirichlet form on a locally compact state space is regular, it is possible to construct an associated Markov process with right continuous sample paths. By the 1980s, the demand for tools to study Markov processes on infinite dimensional (not locally compact) spaces led to various extensions of Fukushima's result. In 1992, the general question was settled by the characterization of $\mathrm{Ma}$ and Röckner to the extent that a Dirichlet form on a separable metric space is 
associated with a Markov process with decent sample paths if and only if the form is quasi-regular. This result provides a method to construct Markov processes on separable metric spaces.

The papers in the thesis are concerned with Dirichlet forms of type

$$
\mathcal{E}(F, G)=\int\langle D F, A D G\rangle_{\mathbb{H}} d \nu
$$

Here the diffusion operator is given by

$$
A=\sum_{i=1}^{\infty} \lambda_{i}\left\langle S_{i},\right\rangle_{\mathbb{H}} S_{i}
$$

$S_{i}, i \in \mathbb{N}$ are the Schauder functions, $\mathbb{H}$ is the Cameron-Martin space $C_{0}\left([0,1] ; \mathbb{R}^{d}\right)$ and $\nu$ is the Wiener measure. In the paper [Driver and Röckner, 1992] the classical quasiregular Dirichlet form, i.e. $\lambda_{i}=1, i \in \mathbb{N}$, is studied and and associated with a diffusion process on a compact Riemannian manifold. Similar results but in the case of unbounded diffusion where studied in [Löbus, 2004].

\subsection{Paper I specifics}

In the first paper we investigate the case where the Wiener measure $\nu$ is replaced with the weighted Wiener measure $\varphi \nu$. Here the weight functions $\varphi$ is of the form

$$
\varphi(\gamma)=\exp \left\{\int_{0}^{1}\left\langle b\left(\gamma_{s}\right), d \gamma_{s}\right\rangle_{\mathbb{R}^{d}}-\frac{1}{2} \int_{0}^{1}\left|b\left(\gamma_{s}\right)\right|^{2} d s\right\} .
$$

This particular choice of weight functions is motivated by the geometric setting in the papers [Wang and $\mathrm{Wu}, 2008$, Wang and $\mathrm{Wu}, 2009$ ] by F.-Y. Wang and B. Wu. These weight functions are considered both in a geometric framework and in the flat case. We study the form (1) on the set of smooth cylindrical functions of type

$$
F, G \in Y=\left\{F(\gamma)=f\left(\gamma\left(s_{1}\right), \ldots, \gamma\left(s_{k}\right)\right): s_{j} \text { is a dyadic point }\right\}
$$

and

$$
F, G \in Z=\left\{F(\gamma)=f\left(\gamma\left(s_{1}\right), \ldots, \gamma\left(s_{k}\right)\right): s_{j} \in[0,1]\right\}
$$

Above, $\gamma$ is a Wiener trajectory. In the paper we formulate necessary and sufficient conditions on $\lambda_{1}(\gamma), \lambda_{2}(\gamma), \ldots$ that guarantee closability of $(\mathcal{E}, Y)$ and $(\mathcal{E}, Z)$ on $L^{2}(\varphi \nu)$. The paper then shows locality, Dirichlet property, and quasi-regularity of the closure $(\mathcal{E}, Z)$ on $L^{2}(\varphi \nu)$. The final parts of the paper are devoted to transferring the results into a geometric setting. In this case we consider Wiener trajectories on a stochastically complete Riemannian manifold, a generalization of the results of [Driver and Röckner, 1992] and [Löbus, 2004] where compact manifolds are studied. 


\subsection{Paper II specifics}

The second paper focuses on the process associated with the Dirichlet form (1). In Paper II the diffusion coefficients $\lambda_{i}$ of the diffusion operator (2) are considered to be real numbers. We show that the associated stochastic process has the representation

$$
X_{t}:=\sum_{i=1}^{\infty} G_{i}\left(\lambda_{i} t\right) \cdot S_{i}, \quad t \geq 0,
$$

where, for $t \geq 0$, the right-hand side converges in $C_{0}\left([0,1] ; \mathbb{R}^{d}\right)$. Here $G_{i}, i \in \mathbb{N}$, are independent one-dimensional Ornstein-Uhlenbeck processes, i.e. $d G_{i}(t)=-G_{i}(t) d t+$ $\sqrt{2} d W_{i}(t), t \geq 0$, for a sequence of independent one-dimensional Wiener processes $W_{i}, i \in \mathbb{N}$. We show that the distributions of a sequence of certain finite dimensional Ornstein-Uhlenbeck processes converge weakly to the distribution of the infinite dimensional Ornstein-Uhlenbeck process, $X$, associated with the Dirichlet form $(\mathcal{E}, D(\mathcal{E}))$. We also investigate the quadratic variation for this process, both in the classical sense and in the recent framework of stochastic calculus via regularization. Since the process is Banach space valued, the tensor quadratic variation is an appropriate tool to establish the Itô formula for the process $X$. Sufficient conditions are presented for the scalar as well as the tensor quadratic variation to exist.

\section{Malliavin calculus}

The French mathematician Paul Malliavin developed a calculus that is extending the calculus of variations, from functions to stochastic processes. Among other results it makes it possible to calculate the derivative of random variables. The material in this section is based on David Nualart's monographs [Nualart, 2009] and [Nualart, 2006] and can also be found in [Karlsson, 2013].

\subsection{General framework}

We let $\mathbb{H}$ be a real separable Hilbert space with inner product $\langle\cdot, \cdot\rangle_{\mathbb{H}}$ and corresponding norm $\|\cdot\|_{\mathbb{H}}$. Unless otherwise stated, in this presentation we may consider the Hilbert space $\mathbb{H}$ to be $L^{2}([0,1])$ with the Borel $\sigma$-algebra $\mathcal{B}$ and Lebesgue measure $\mu$.

Definition 2.1. A stochastic process $W=\{W(h), h \in \mathbb{H}\}$ defined in a complete probability space $(\Omega, \mathcal{F}, \mathbb{P})$ is a Gaussian process on $\mathbb{H}$, if $W$ is a centered Gaussian family of random variables and $E[W(g) W(h)]=\langle g, h\rangle_{\mathbb{H}}$ for all $g, h \in \mathbb{H}$.

This can be illustrated by a simple example.

Example .1

Let $\mathbb{H}=L^{2}(\mathbb{R}+)$ and $W_{t}:=W(\mathbb{1}[0, t]), t \geq 0$. This is standard Brownian motion since

$$
E\left[W_{s} W_{t}\right]=E\left[W\left(\mathbb{1}_{[0, s]}\right) W\left(\mathbb{1}_{[0, t]}\right)\right]=\left\langle\mathbb{1}_{[0, s]}, \mathbb{1}_{[0, t]}\right\rangle_{L^{2}}=\min (s, t),
$$

i.e. the covariance function for Brownian motion. 


\subsection{Hermite polynomials}

The Hermite polynomials will play an important role in the upcoming Wiener chaos decomposition of a Gaussian random variable as it turns out they have certain orthogonality properties. Here we define the Hermite polynomials $H_{n}$ as the coefficients of the Taylor expansion of $F(x, t)=\exp \left\{t x-t^{2} / 2\right\}$ in powers of $t$. In other words

$$
F(x, t):=e^{t x-\frac{t^{2}}{2}}=\sum_{n=0}^{\infty} H_{n}(x) \cdot t^{n} .
$$

We have the relations

$$
H_{0}(x)=1, \quad H_{1}(x)=x, \quad H_{2}(x)=\frac{1}{2}\left(x^{2}-1\right), \quad H_{n}(x)=\frac{(-1)^{n}}{n !} e^{\frac{x^{2}}{2}} \frac{d^{n}}{d x^{n}}\left(e^{-\frac{x^{2}}{2}}\right)
$$

and also

$$
\frac{d}{d x} H_{n}(x)=H_{n-1}(x), \quad(n+1) H_{n+1}(x)=x H_{n}(x)-H_{n-1}(x)
$$

as well as

$$
H_{n}(0)= \begin{cases}0 & \text { if } n \text { odd } \\ \frac{(-1)^{k}}{2^{k} k !} & \text { if } n \text { even }\end{cases}
$$

\subsection{Wiener Chaos}

Assume that $\mathbb{H}$ (we may think of it as $L^{2}([0,1])$ ) is infinite dimensional and let $\left\{e_{1}, e_{2}, \ldots\right\}$ be an ON-basis in $\mathbb{H}$. We let $\Lambda$ denote the set of all finite multi-indices $a=\left(a_{1}, a_{2}, \ldots\right)$, $a_{i} \in \mathbb{N}$ for $i=1,2, \ldots$, i.e. only finitely many $a_{i}$ :s are non-zero. For any $a \in \Lambda$ we define

$$
a !:=\prod_{i=1}^{\infty} a_{i} !, \quad|a|:=\sum_{i=1}^{\infty} a_{i}, \quad \Phi_{a}:=\sqrt{a !} \prod_{i=1}^{\infty} H_{a_{i}}\left(W\left(e_{i}\right)\right) .
$$

Taking the Hermite polynomials of Gaussian variables we obtain an orthogonality relation in the following way. For a two-dimensional Gaussian vector $(X, Y)$ s.t. $E[X]=E[Y]=$ 0 and $V[X]=V[Y]=1$, we have that for all $m, n \in \mathbb{N}$

$$
E\left[H_{m}(X) H_{n}(Y)\right]= \begin{cases}0 & \text { if } m \neq n, \\ \frac{1}{n !} E[X Y]^{n} & \text { if } m=n .\end{cases}
$$

Let $\mathcal{H}_{n}$ denote the closed subspace of $L^{2}(\Omega, \mathcal{F}, \mathbb{P})$ spanned by $\left\{\Phi_{a}: a \in \Lambda,|a|=n\right\}$. It can be shown that $L^{2}(\Omega, \mathcal{F}, \mathbb{P})=\bigoplus_{n=0}^{\infty} \mathcal{H}_{n}$. We call $\mathcal{H}_{n}$ the $n$th Wiener Chaos. Furthermore we let $J_{n}$ denote the projection operator onto the $n$th Wiener Chaos. It follows for $F \in L^{2}(\Omega, \mathcal{F}, \mathbb{P})$ that

$$
F=\sum_{n=0}^{\infty} J_{n} F
$$

Remark 2.2. The $n$th Wiener chaos contains polynomials of Gaussian variables of degree $n$.

While we defined $n$th Wiener chaos as an expression of ON-basis $\left(e_{i}\right)_{i=1}^{\infty}$, it turns out that the $n$th Wiener chaos is independent of this choice of ON-basis. 


\subsection{Multiple stochastic integrals}

We will see that there is another representation for the $n$th Wiener chaos in the form of iterated Itô integrals. We begin with introducing a type of elementary functions first described by Norbert Wiener.

Definition 2.3. We define $\mathbb{E}_{n}$ as the set of all elementary functions of form

$$
f\left(t_{1}, \ldots, t_{n}\right)=\sum_{i_{1}, \ldots, i_{n}=1}^{k} a_{i_{1} \ldots i_{n}} \mathbb{1}_{A_{i_{1}} \times \cdots \times A_{i_{n}}}\left(t_{1}, \ldots, t_{n}\right),
$$

where $a_{i_{1} \ldots i_{n}} \in \mathbb{R}$ and $a_{i_{1} \ldots i_{n}}=0$ if two indices coincide and $A_{1}, \ldots, A_{k}$ are pairwise disjoint subsets of $[0,1]$ with finite Lebesgue measure.

We see that this is the usual simple functions in $[0,1]^{n}$ with the condition of being 0 on any diagonal described by two equal coordinates. It can be shown that this set has measure 0 and thus the set of elementary functions $\mathbb{E}_{n}$ is dense in $L^{2}\left([0,1]^{n}\right)$. We can now define a stochastic integral for these elementary functions. For a function of the form (4) we define

$$
I_{n}\left(f\left(t_{1}, \ldots, t_{n}\right)\right)=\sum_{i_{1}, \ldots, i_{n}=1}^{k} a_{i_{1}, \ldots, i_{n}} W\left(A_{i_{1}}\right) \cdot \ldots \cdot W\left(A_{i_{n}}\right) .
$$

Remark 2.4. $I_{n}(f)$ can be expressed as an iterated Itô integral

$$
I_{n}(f)=n ! \int_{t_{n}=0}^{1} \int_{t_{n-1}=0}^{t_{n}} \ldots \int_{t_{1}=0}^{t_{2}} f\left(t_{1}, \ldots, t_{n}\right) d W_{t_{1}} \ldots d W_{t_{n}} .
$$

The following proposition shows some of the properties of this stochastic integral.

Proposition 2.5. $I_{n}$ has the following properties:

(i) $I_{n}(f)=I_{n}(\tilde{f})$, where $\tilde{f}$ denotes the symmetrization of $f$, i.e.

$$
\tilde{f}\left(t_{1}, \ldots, t_{n}\right)=\frac{1}{n !} \sum_{\Pi} f\left(t_{\Pi(1)}, \ldots, t_{\Pi(n)}\right)
$$

where $\Pi$ runs over all permutations of $\{1, \ldots, n\}$.

(ii)

$$
E\left[I_{m}(f), I_{n}(g)\right]= \begin{cases}0 & \text { if } m \neq n \\ n !\langle\tilde{f}, \tilde{g}\rangle_{L^{2}\left([0,1]^{n}\right)} & \text { if } m=n .\end{cases}
$$

It follows that we get an isometry between $L_{s}^{2}\left([0,1]^{n}\right):=\left\{\tilde{f}: f \in L^{2}\left([0,1]^{n}\right)\right\}$ and $\mathcal{H}_{n}$, i.e.

$$
\sqrt{n !}\|\tilde{f}\|_{L^{2}\left([0,1]^{n}\right)}=\|I(f)\|_{L^{2}(\Omega, \mathcal{F}, \mathbb{P})} .
$$

Since $\mathbb{E}_{n}$ is dense in $L^{2}\left([0,1]^{n}\right)$ we can extend $I_{n}$ to a linear continuous operator from $L^{2}\left([0,1]^{n}\right)$ to $L^{2}(\Omega, \mathcal{F}, \mathbb{P})$. 
Remark 2.6. The image of $L_{s}^{2}\left([0,1]^{n}\right)$ under $I_{n}$ is the $n$th Wiener chaos $\mathcal{H}_{n}$ since $I_{n}(f)$ is a polynomial of degree $n$ in $W\left(A_{1}\right), \ldots, W\left(A_{n}\right)$ when $f$ has the form (4), and Proposition 2.5(ii) shows that stochastic integrals of different order are orthogonal. The claim then follows from induction.

Since we can express a random variable in its chaos expansion we note that for $F \in$ $L^{2}(\Omega, \mathcal{F}, \mathbb{P})$, there exists $f_{n} \in L_{s}^{2}\left([0,1]^{n}\right), n \in \mathbb{N}$ such that

$$
F=E[F]+\sum_{n=1}^{\infty} I_{n}\left(f_{n}\right) \text {. }
$$

\subsection{Derivative operator}

To define the calculus on the Wiener space a natural way of proceeding is to first look at a simple class of functions. Let $Z$ denote the set of smooth cylindrical functions

$$
Z=\left\{F: F=f\left(W\left(h_{1}\right), \ldots, W\left(h_{n}\right)\right), h_{1}, \ldots, h_{n} \in \mathbb{H}, f \in C_{p}^{\infty}\right\}
$$

where $C_{p}^{\infty}$ denotes smooth functions with polynomial growth.

Definition 2.7. The gradient of a smooth cylindrical function is defined by:

$$
D F:=\sum_{n=1}^{n} \frac{\partial f}{\partial x_{i}}\left(W\left(h_{1}\right), \ldots, W\left(h_{n}\right)\right) \cdot h_{i} .
$$

Remark 2.8. We have that $D W(h)=h$.

Clearly we have the product rule $D(F G)=D F \cdot G+F \cdot D G$ and we also get an integration by parts formula as will be shown shortly. We also define the directional derivative in the following manner.

Definition 2.9. The directional derivative $D_{h}$ is defined by

$$
D_{h} F:=\langle D F, h\rangle \quad h \in \mathbb{H} .
$$

Proposition 2.10. Let $F \in \mathbb{D}^{1,2}$ and assume that $F$ has representation

$$
F=E[F]+\sum_{n=1}^{\infty} I_{n}\left(f_{n}\right)
$$

where $f_{n} \in L_{s}^{2}\left([0,1]^{n}\right)$. Then

$$
D_{t} F=\sum_{n=1}^{\infty} n I_{n-1}\left(f_{n}(\cdot, t)\right), \quad t \in[0,1] .
$$

Proof: First let $F=I_{n}\left(f_{n}\right)$ where

$$
f_{n}\left(t_{1}, \ldots, t_{n}\right)=\sum_{i_{1}, \ldots, i_{n}}^{k} a_{i_{1} \ldots i_{n}} \mathbb{1}_{A_{i_{1}} \times \cdots \times A_{i_{n}}}\left(t_{1}, \ldots, t_{n}\right) .
$$


Then

$$
F=I_{n}\left(f_{n}\right)=\sum_{i_{1}, \ldots, i_{n}=1}^{k} a_{i_{1} \ldots i_{n}} W\left(A_{i_{1}}\right) \cdot \ldots \cdot W\left(A_{i_{n}}\right)
$$

and $F \in Z$. We get

$$
D_{t} F=\sum_{j=1}^{n} \sum_{i_{1}, \ldots, i_{n}=1}^{k} a_{i_{1} \ldots i_{n}} W\left(A_{i_{1}}\right) \cdot \ldots \cdot \mathbb{1}_{A_{i_{j}}}(t) \cdot \ldots \cdot W\left(A_{i_{n}}\right)=n I_{n-1}(f(\cdot, t))
$$

by symmetry. The claim follows from the closedness of $\mathbb{D}^{1,2}$.

\subsection{Divergence operator}

We introduce the divergence operator $\delta$. It turns out that $\delta$ is the adjoint operator to $D$. Let Dom $\delta$ be the set of all $u \in L^{2}(\Omega, \mathcal{F}, \mathbb{P} ; \mathbb{H})$ such that there exists $c(u)>0$ with

$$
\left|E\left[\langle D F, u\rangle_{\mathbb{H}}\right]\right| \leq c\|F\|_{L^{2}}
$$

for all $F \in \mathbb{D}^{1,2}$. Then $E\left[\langle D F, u\rangle_{\mathbb{H}}\right]$ is a bounded linear functional from $\mathbb{D}^{1,2}$ to $\mathbb{R}$. Now since $\mathbb{D}^{1,2}$ is dense in $L^{2}(\Omega, \mathcal{F}, \mathbb{P})$ Riesz representation theorem states that there exists a unique representing element $\delta(u)$, bounded in $L^{2}(\Omega, \mathcal{F}, \mathbb{P})$. That is

$$
E\left[\langle D F, u\rangle_{\mathbb{H}}\right]=E[F \delta(u)], \quad \forall F \in \mathbb{D}^{1,2}
$$

Just like for the derivative there is a connection between the Wiener chaos expansion and the divergence operator. Recall that for $u \in L^{2}([0,1] \times \Omega), u$ has a Wiener chaos expansion of form

$$
u(t)=\sum_{n=0}^{\infty} I_{n}\left(f_{n}(\cdot, t)\right)
$$

where $f_{n} \in L^{2}\left([0,1]^{n}\right)$ and $f_{n}$ is symmetric in the $n$ first variables.

Proposition 2.11. We have $u \in$ Dom $\delta$ if and only if the series

$$
\sum_{n=0}^{\infty} I_{n+1}\left(\tilde{f}_{n}\right)
$$

converges in $L^{2}(\Omega, \mathcal{F}, \mathbb{P})$. In this case we have

$$
\delta(u)=\sum_{n=0}^{\infty} I_{n+1}\left(\tilde{f}_{n}\right)
$$


Proof: Suppose $G=I_{n}(g)$ where $g \in L_{s}^{2}\left([0,1]^{n}\right)$. Now using Proposition 2.10 we get

$$
\begin{aligned}
E\left[\langle u, D G\rangle_{\mathbb{H}}\right] & =E\left[\left\langle u, n I_{n-1}(g(\cdot, t))\right\rangle_{\mathbb{H}}\right]=E\left[\left\langle I_{n-1}\left(f_{n-1}(\cdot, t)\right), n I_{n-1}(g(\cdot, t))\right\rangle_{\mathbb{H}}\right] \\
& =\int_{[0,1]} E\left[I_{n-1}\left(f_{n-1}(\cdot, t)\right) n I_{n-1}(g(\cdot, t))\right] d t \\
& =n(n-1) ! \int_{[0,1]}\left\langle f_{n-1}, g\right\rangle_{L^{2}\left([0,1]^{n-1}\right)} d t \\
& =n !\left\langle f_{n-1}, g\right\rangle_{L^{2}\left([0,1]^{n}\right.}=n !\left\langle\tilde{f}_{n-1}, g\right\rangle_{L^{2}\left([0,1]^{n}\right)} \\
& =E\left[I_{n}\left(\tilde{f}_{n-1}\right) I_{n}(g)\right]=E\left[I_{n}\left(\tilde{f}_{n-1}\right) G\right]
\end{aligned}
$$

where the third and last lines come from the isometry between $L_{s}^{2}([0,1])^{n}$ and $\mathcal{H}_{n}$.

One can see from this that $\delta$ is an integral. $\delta(u)$ is called the Skorokhod stochastic integral of the process $u$ and if the process is adapted then the Skorokhod integral will coincide with the Itô integral, i.e.

$$
\delta(u)=\int_{0}^{1} u_{t} d W_{t} .
$$

\section{Dirichlet forms}

The theory of Dirichlet forms was first introduced in the works by Beurling and Deny in 1958 and 1959. Dirichlet form theory can be used in the area of Markov processes giving a different approach to problems. The usual tools for studying diffusion theory are methods from partial differential equations, whereas Dirichlet forms are connected to potential theory and energy methods. This section is based on the book [Ma and Röckner, 1992] which contains a much more through treatment of the subject.

Let $\mathbb{H}$ be a Hilbert space with corresponding norm $\|\cdot\|_{\mathbb{H}}$ and inner product $\langle\cdot, \cdot\rangle_{\mathbb{H}}$.

Definition 3.1. A Dirichlet form is a densely defined positive symmetric bilinear form on $L^{2}(E, \mu)$ such that

(i) $D(\mathcal{E})$ is a real Hilbert space with inner product $\langle u, v\rangle_{\mathcal{E}}:=\langle,\rangle_{E}^{2}+\mathcal{E}(u, v)^{2}$,

(ii) For every $u \in D(\mathcal{E})$ it holds $u^{+} \wedge 1 \in D(\mathcal{E})$ and $\mathcal{E}\left(u^{+} \wedge 1, u^{+} \wedge 1\right) \leq \mathcal{E}(u, u)$.

Not all Dirichlet forms have an associated process. What follows are the different notions needed for the formulation of Ma-Röckner's theorem.

Definition 3.2. Let $\mathbb{H}=L^{2}(E, m)$. A bilinear form $\mathcal{E}$ on $E$ is regular if $D(\mathcal{E}) \cap C_{0}(E)$ is dense in $D(\mathcal{E})$ w.r.t. $\mathcal{E}_{1}$-norm and dense in $C_{0}(E)$ w.r.t. the uniform norm. Here $C_{0}(E)$ denotes all continuous functions on $E$ with compact support.

Next we recall some basic definitions from the theory of stochastic processes. 
Definition 3.3. We say that a function is cadlag if it is right continuous with left limits, i.e.

(i) $f(x-):=\lim _{t \nearrow x} f(t) \quad$ exists,

(ii) $f(x+):=\lim _{t \searrow x} f(t) \quad$ exists and is equal to $f(x)$.

The corresponding term for left continuous functions is caglad.

Definition 3.4. Two stochastic processes $X$ and $Y$ with a common index set $T$ are called versions of one another if

$$
t \in T, P(\{\omega: X(t, \omega)=Y(t, \omega)\})=1 .
$$

Such processes are also said to be stochastically equivalent.

If in addition the process $X_{t}$ is left or right continuous then for a version $Y_{t}$ we have $X_{t}=Y_{t}$ almost surely.

Definition 3.5. (i) An increasing sequence $\left(F_{k}\right)_{k \in \mathbb{N}}$ of closed subsets of $E$ is called an $\mathcal{E}$-nest if $\cup_{k \geq 1} D(\mathcal{E})_{F_{k}}$ is dense in $D(\mathcal{E})$ w.r.t. $\|\cdot\|_{\tilde{\varepsilon}_{1}}$, where $D(\mathcal{E})_{F_{k}}$ denotes $\{u \in D(\mathcal{E})$ : $u=0 m$-a.e. on $\left.E \backslash F_{k}\right\}$.

(ii) A subset $N \subset \mathcal{E}$ is called $\mathcal{E}$-exceptional if $N \subset \cap_{k \geq 0} F_{k}^{c}$ for some $\mathcal{E}$-nest $\left(F_{k}\right)_{k \in \mathbb{N}}$. (iii) We say that a property holds $\mathcal{E}$-quasi-everywhere if it holds everywhere outside some $\mathcal{E}$-exceptional set.

We can relate the definition of quasi-continuity to a similar notion on the $\mathcal{E}$-nest.

Definition 3.6. An $\mathcal{E}$-quasi-everywhere defined function $f$ is called $\mathcal{E}$-quasi-continuous if there exists an $\mathcal{E}$-nest $\left(F_{k}\right)_{k \in \mathbb{N}}$ such that $f$ is continuous on $\left(F_{k}\right)_{k \in \mathbb{N}}$.

Definition 3.7. A Dirichlet form $(\mathcal{E}, D(\mathcal{E}))$ on $L^{2}(E, m)$ is called quasi-regular if:

(i) There exists an $\mathcal{E}$-nest $\left(F_{k}\right)_{k \in \mathbb{N}}$ consisting of compact sets,

(ii) There exists an $\tilde{\mathcal{E}}_{1}^{1 / 2}$-dense subset of $D(\mathcal{E})$ whose elements have $\mathcal{E}$-quasi-continuous $m$-versions,

(iii) There exist $u_{n} \in D(\mathcal{E}), n \in \mathbb{N}$, having $\mathcal{E}$-quasi-continuous $m$-versions $\tilde{u}_{n}$, $n \in \mathbb{N}$, and an $\mathcal{E}$-exeptional set $N \subset E$ such that $\left\{\tilde{u}_{n}: n \in \mathbb{N}\right\}$ separates the points of $E \backslash N$.

Definition 3.8. A process $M$ with state space $E$ is called $\mu$-tight if there exists an increasing sequence $\left(K_{n}\right)_{n \in \mathbb{N}}$ of compact sets in $E$ such that

$$
P_{\mu}\left(\lim _{n \rightarrow \infty} \inf \left\{t>0: M_{t} \in E \backslash K_{n}\right\}<\infty\right)=0
$$

where inf $\varnothing:=\infty$.

We note that it simply means that for every $\varepsilon>0$ there exists a compact set $K$ such that $P_{\mu}\left(M_{t} \in K\right)>1-\varepsilon$. 
Definition 3.9. A cadlag Markov process $M$ with state space $E$ and transition semigroup $\left(p_{t}\right)_{t>0}$ is said to be properly associated with $(\mathcal{E}, D(\mathcal{E}))$ and its corresponding semigroup $\left(T_{t}\right)_{t>0}$ if $p_{t} f$ is an $\mathcal{E}$-quasi continuous $\mu$-version of $T_{t} f$ for all $t>0$ and all bounded $f \in L^{2}(E ; \mu)$.

The following theorem is the main purpose of this section. It provides the connection between Dirichlet forms and process theory.

Theorem 3.10. Let $(\mathcal{E}, D(\mathcal{E}))$ be a quasi-regular Dirichlet form on $L^{2}(E, \mu)$. Then there exists a pair $(M, \hat{M})$ of $\mu$-tight special standard processes which is properly associated with $(\mathcal{E}, D(\mathcal{E}))$.

Proof: The proof is omitted but can be found in [Ma and Röckner, 1992].

Remark 3.11. It is known that if $E$ is locally compact, i.e. finite dimensional, then there exists an associated process to every regular Dirichlet form on $L^{2}(E, \mu)$. See [Fukushima et al., 2011].

\section{Stochastic integration via regularization}

This section contains some notions in the area of stochastic integration via regularization. Stochastic integration via regularization is a recently developed theory using techniques from integration. The different objects in this theory are presented first for finite dimensional stochastic processes, before considering the corresponding objects in the infinite dimensional case. This section is based on material in [Russo and Vallois, 2007], [Di Girolami and Russo, 2014] and [Di Girolami et al., 2014].

\subsection{One dimensional case}

In this section let $X(t), t \geq 0$, be a real-valued continuous process and $Y(t), t \geq 0$, be a real-valued locally integrable process. Below we introduce the key concept of uniform convergence in probability, abbreviated ucp.

Definition 4.1. A sequence of real-valued processes $\left(X_{t}^{\delta}\right)_{t \in[0, T]}$ is said to converge to $\left(X_{t}\right)_{t \in[0, T]}$ in the ucp sense as $\delta \rightarrow 0$, if for all $\varepsilon>0$

$$
\lim _{\delta \rightarrow 0} P\left(\sup _{t \in[0, T]}\left|X_{t}^{\delta}-X_{t}\right|>\varepsilon\right)=0 .
$$

We may now introduce the following integral and covariation.

Definition 4.2. Provided that the following limits exist in the ucp sense we define the forward integral

$$
\int Y d^{-} X:=\lim _{\delta \rightarrow 0} \int_{0}^{t} Y_{s} \frac{X_{s+\delta}-X_{s}}{\delta} d s
$$


and the covariation

$$
[X, Y]_{t}:=\lim _{\delta \rightarrow 0} \int_{0}^{t} \frac{\left(X_{s+\delta}-X_{s}\right)\left(Y_{s+\delta}-Y_{s}\right)}{\delta} d s .
$$

We also denote $[X]_{t}:=[X, X]_{t}$ and call it the quadratic variation of $X$.

Remark 4.3. In e.g. the case of $Y$ being continuous with bounded variation then the forward integral coincides with the usual Itô integral, see [Russo and Vallois, 2007].

In the one dimensional case we have the following Itô formula from [Russo and Vallois, 2007] Proposition 12.

Proposition 4.4. Suppose that $[X]_{t}, t \geq 0$ exists and $f \in C^{2}(\mathbb{R})$. Then $\int f^{\prime}\left(X_{s}\right) d^{-} X_{s}$ exists and

$$
f\left(X_{t}\right)=f\left(X_{0}\right)+\int_{0}^{t} f^{\prime}\left(X_{s}\right) d^{-} X_{s}+\frac{1}{2} \int_{0}^{t} f^{\prime \prime}\left(X_{s}\right) d[X]_{s} .
$$

\subsection{Infinite dimensional case}

We now consider the corresponding notions in the infinite dimensional case. We now consider $X$ to be a process taking values in a Banach space $B$. Ucp convergence is defined in a similar way as above.

Definition 4.5. A sequence of $B$ valued processes $\left(X_{t}^{\delta}\right)_{t \in[0, T]}$ is said to converge to $\left(X_{t}\right)_{t \in[0, T]}$ in the ucp sense as $\delta \rightarrow 0$, if for all $\varepsilon>0$

$$
\lim _{\delta \rightarrow 0} P\left(\sup _{t \in[0, T]}\left\|X_{t}^{\delta}-X_{t}\right\|_{B}>\varepsilon\right)=0 .
$$

In [Di Girolami and Russo, 2014] Definition 5.1 the authors introduce the following definition.

Definition 4.6. Let $X$ be a $B$ valued process and $Y$ be a $B^{*}$ valued process such that $X$ is continuous and $\int_{0}^{T}\left\|Y_{s}\right\|_{B^{*}} d s<\infty$ a.s.. Provided that the following limit exist in probability for every $t \in[0, T]$ we define the forward integral

$$
\int_{0}^{t} B^{*}\left\langle Y_{s}, d^{-} X_{s}\right\rangle_{B}:=\lim _{\delta \rightarrow 0} \int_{0}^{t}\left\langle Y_{s}, \frac{X_{s+\delta}-X_{s}}{\delta}\right\rangle_{B} d s
$$

if the process

$$
\left(\int_{0}^{t} B^{*}\left\langle Y_{s}, d^{-} X_{s}\right\rangle_{B}\right)_{t \in[0, T]}
$$

admits a continuous version. 
In e.g. the case of $B=L^{1}([0,1])$, the duality pairing $B^{*}\left\langle g^{*}, f\right\rangle_{B}$ has the integral representation

$$
B^{*}\left\langle g^{*}, f\right\rangle_{B}=\int_{0}^{1} f \cdot g d x
$$

for $f \in B=L^{1}([0,1]), g^{*} \in B^{*}$ and some representing element $g \in L^{\infty}([0,1]) \cong B^{*}$. In the case of $F \in B \widehat{\otimes}_{\pi} B \cong L^{1}\left([0,1]^{2} ; \mathbb{R}^{d^{2}}\right), G^{*} \in\left(B \widehat{\otimes}_{\pi} B\right)^{*}$ and some representing element $G \in L^{\infty}\left([0,1]^{2} ; \mathbb{R}^{d^{2}}\right) \cong\left(B \widehat{\otimes}_{\pi} B\right)^{*}$ the pairing duality becomes

$$
\left(B \widehat{\otimes}_{\pi} B\right)^{*}\left\langle G^{*}, F\right\rangle_{\left(B \widehat{\otimes}_{\pi} B\right)^{* *}}=\int F(u, v) \cdot G(u, v) d u d v,
$$

where the symbol $\bullet$ to denotes the scalar product in $\mathbb{R}^{d^{2}}$.

The paper [Di Girolami and Russo, 2014] also defines the following notions of covariation.

Definition 4.7. Let $X$ and $Y$ be a $B_{1}$ respectively $B_{2}$ valued processes. We define the scalar covariation as the ucp limit

$$
[X, Y]_{t}:=\lim _{\delta \rightarrow 0} \int_{0}^{t} \frac{\left\|X_{s+\delta}-X_{s}\right\|_{B_{1}}\left\|Y_{s+\delta}-Y_{s}\right\|_{B_{2}}}{\delta} d s
$$

provided it exists. We also denote $[X]_{t}:=[X, X]_{t}$ and call it the scalar quadratic variation of $X$.

Definition 4.8. Let $X$ and $Y$ be $B_{1}$ respectively $B_{2}$ valued processes. We define the tensor covariation as the ucp limit

$$
[X, Y]_{t}^{\otimes}:=\lim _{\delta \rightarrow 0} \int_{0}^{t} \frac{\left(X_{s+\delta}-X_{s}\right) \otimes\left(Y_{s+\delta}-Y_{s}\right)}{\delta} d s,
$$

provided it exists. We also denote $[X]_{t}^{\otimes}:=[X, X]_{t}^{\otimes}$ and call it the tensor quadratic variation of $X$.

It is worth mentioning that quadratic variation in the sense above is essentially only suitable for semimartingale processes, see [Di Girolami et al., 2014]. However in the situation where a process admits having both scalar and tensor quadratic variation we get the Itô formula in the form of [Di Girolami and Russo, 2014] Theorem 5.2.

Proposition 4.9. Suppose that $X$ is a $B$ valued continuous process admitting a scalar quadratic variation and a tensor quadratic variation. Furthermore let $F$ be a mapping $F:[0, T] \times B \rightarrow \mathbb{R}$ such that $F$ is one time continuously Fréchet differentiable and two times continuously Fréchet differentiable in the second argument. That is, denoting the Fréchet derivative with respect to the second variable by $D$, for every $t \in[0, T]$ we 
have $D F(t, \cdot): B \rightarrow B^{*}$ and $D^{2} F(t, \cdot): B \rightarrow\left(B \widehat{\otimes}_{\pi} B\right)^{*}$ continuously. Under these conditions for every $t \in[0, T]$ the forward integral

$$
\int_{0}^{t} B^{*}\left\langle D F\left(s, X_{s}\right), d^{-} X_{s}\right\rangle_{B} \quad \text { exists }
$$

and

$$
\begin{aligned}
F\left(t, X_{t}\right)= & F\left(0, X_{0}\right)+\int_{0}^{t} \partial_{t} F\left(s, X_{s}\right) d s+\int_{0}^{t} B^{*}\left\langle D F\left(s, X_{s}\right), d^{-} X_{s}\right\rangle_{B} \\
& +\frac{1}{2} \int_{0}^{t}\left(B \widehat{\otimes}_{\pi} B\right)^{*}\left\langle D^{2} F\left(s, X_{s}\right), d[X]_{s}\right\rangle_{\left(B \widehat{\otimes}_{\pi} B\right)^{* *}} \cdot
\end{aligned}
$$

Remark 4.10. The above proposition makes use of the existence of scalar quadratic variation and a tensor quadratic variation. The statement then follows as a direct consequence of [Di Girolami et al., 2014] Remark 5.7 applied to the mentioned Theorem 5.2 of [Di Girolami and Russo, 2014].

\section{The geometric Cameron-Martin formula}

This chapter serves to introduce the concepts needed for construction of Brownian motion on a manifold $M$. The main part of section is based on the series of lecture notes [Löbus, 1995]. Much of the material can also be found in [Rogers and Williams, 2000]. We first recall some basic definitions to introduce the notation used.

\subsection{Manifolds}

Definition 5.1. A subset $M \subset \mathbb{R}^{N}$ is called a $d$-dimensional $C^{\infty}$-manifold in $\mathbb{R}^{N}$ if for each $x \in M$ there is an open (in the relative topology) set $G \subset M$ with $x \in G$ and there exist $F \in C^{\infty}\left(\mathbb{R}^{d} \longrightarrow \mathbb{R}^{N-d}\right)$ such that (if necessary, after a permutation of coordinates)

$$
G=\{(y, F(y)): y \in A\},
$$

for an open set $A \subset \mathbb{R}^{d}$.

Definition 5.2. We say that $y(t), t \in[0,1]$ is a smooth curve in $\mathbb{R}^{d}$ if

$$
y(t)=\sum_{i=1}^{d} y^{i}(t) e_{i}, \quad t \in[0,1],
$$

where $e_{i}=\left(0, \ldots, 0,{ }_{i}, 0, \ldots, 0\right), y^{i} \in C^{\infty}([0,1]), i=1, \ldots, d$, such that

$$
\sum_{i=1}^{d}\left(\dot{y}^{i}(t)\right)^{2} \neq 0, \quad t \in[0,1] .
$$


With the notation used in the previous definition, $(x(t), t \in[0,1])$ defined as

$$
x(t):=(y(t), F(y(t))), \quad t \in[0,1],
$$

is called a smooth curve on $M$.

We recall the definition of tangents and tangent space.

Definition 5.3. We say that $v(t)=\dot{y}(t)$ is a tangent to $(y(s), s \in[0,1])$ (at $t$ ). Let $\mathrm{I}_{\mathrm{d}}$ denote the $d \times d$ identity matrix and $J(y)$ denote the Jacobian matrix

$$
\left(J_{i}^{r}(y)\right)_{i, r=1, \ldots, d}=\left(\frac{\partial F^{r}}{\partial y^{i}}\right)_{i, r=1, \ldots, d} .
$$

We use the notation $\left(\mathrm{I}_{\mathrm{d}}, J\right)^{T}$ for the matrix

$$
\left[\begin{array}{ccc}
1 & \cdots & 0 \\
& \ddots & \\
0 & \cdots & 1 \\
\frac{\partial F^{1}}{\partial y^{1}} & \cdots & \frac{\partial F^{1}}{\partial y^{d}} \\
\vdots & & \vdots \\
\frac{\partial F^{n}}{\partial y^{1}} & \cdots & \frac{\partial F^{n}}{\partial y^{d}}
\end{array}\right]
$$

We call

$$
u(t):=\dot{x}(t)=\left(\mathrm{I}_{\mathrm{d}}, J(y(t))\right)^{T} v(t)
$$

a tangent to $(x(s), s \in[0,1])$ at $t$.

Definition 5.4. For a fixed $y \in \mathbb{R}^{d}$ and the corresponding point $x=(y, F(y)) \in M$. We define $\mathbf{T}_{x}:=\left\{x+t: x \in M, t=\left(\mathrm{I}_{\mathrm{d}}, J(y)\right)^{T} v\right.$ for some $\left.v \in \mathbb{R}^{d}\right\}$ which we identify with all the tangents to $M$ at the point $x$ and call it the tangentspace to $M$ at $x$.

We may induce a metric on the tangent space from the Euclidean metric in $\mathbb{R}^{N}$. Let $u_{1}, u_{2} \in \mathbf{T}_{x}$ where $x=(y, F(y)), y \in \mathbb{R}^{d}$, and $v_{1}, v_{2} \in \mathbb{R}^{d}$ where $u_{1}=\left(\mathrm{I}_{\mathrm{d}}, J(y)\right)^{T} v_{1}$, $u_{2}=\left(\mathrm{I}_{\mathrm{d}}, J(y)\right)^{T} v_{2}$. Then

$$
\left\langle u_{1}, u_{2}\right\rangle_{\mathbb{R}^{N}}=v_{1}^{T}\left(\mathrm{I}_{\mathrm{d}}, J(y)\right)\left(\mathrm{I}_{\mathrm{d}}, J(y)\right)^{T} v_{2} .
$$

We introduce the notation

$$
g(y):=\left(\mathrm{I}_{\mathrm{d}}, J(y)\right)\left(\mathrm{I}_{\mathrm{d}}, J(y)\right)^{T}=\mathrm{I}_{\mathrm{d}}+J(y) J(y)^{T}
$$

and write (8) as

$$
\left\langle u_{1}, u_{2}\right\rangle_{\mathbf{T}_{x}}=v_{1}^{T} g(y) v_{2}=: g\left\langle v_{1}, v_{2}\right\rangle .
$$

Remark 5.5. The length of a smooth curve $(x(t), t \in[0,1])$ on $M$ is calculated by

$$
\int_{0}^{1}\left(\dot{y}(t)^{T} g(y(t)) \dot{y}(t)\right)^{1 / 2} d t
$$

where $x(t)=(y(t), F(y(t))), t \in[0,1]$. 
We now recall the notion of parallel transport along a curve.

Definition 5.6. For a smooth curve $(y(s), s \in[0,1])$ in $\mathbb{R}^{d}$ and the corresponding smooth curve $(x(s), s \in[0,1])$ on $M$, i.e. $x(s)=(y(s), F(y(s))), s \in[0,1]$, let $t_{1}, t_{2} \in[0,1]$ and $u\left(t_{1}\right) \in \mathbf{T}_{x\left(t_{1}\right)}$. Assume $0 \leq t_{1} \leq t_{2} \leq 1$ and let $v(t) \in \mathbf{T}_{x(t)}$ such that $w(t) \in \mathbb{R}^{d}$, $t \in\left[t_{1}, t_{2}\right]$ and

$$
v(t)=\left(\mathrm{I}_{\mathrm{d}}, J(y(t))\right)^{T} w(t), \quad t \in\left[t_{1}, t_{2}\right] .
$$

Let $\dot{v}(t) \in \mathbb{R}^{N}$ denote the rate of change of $v(t)$ and let $P_{x(t)}(\cdot)$ denote the orthogonal projection onto the tangent space $\mathbf{T}_{x(t)}$. We say that $v(t)$ moves parallel in $t \in\left[t_{1}, t_{2}\right]$, along $x(s)$, if for all $t \in\left[t_{1}, t_{2}\right]$ we have $P_{x(t)} \dot{v}(t)=0$.

Remark 5.7. In this case we have

$$
P_{x(t)}(\cdot)=\left(\mathrm{I}_{\mathrm{d}}, J(y(t))\right)^{T} g^{-1}(y(t))\left(\mathrm{I}_{\mathrm{d}}, J(y(t))\right)(\cdot),
$$

since for all $w \in \mathbf{T}_{x(t)}$ and all $z \in \mathbb{R}^{N}$ we have for $w=\left(\mathrm{I}_{\mathrm{d}}, J(y(t))\right)^{T} q$

$$
\begin{aligned}
\left\langle P_{x(t)} z, w\right\rangle_{\mathbb{R}^{N}} & =\left\langle\left(\mathrm{I}_{\mathrm{d}}, J\right)^{T} g^{-1}\left(\mathrm{I}_{\mathrm{d}}, J\right) z,\left(\mathrm{I}_{\mathrm{d}}, J\right)^{T} q\right\rangle_{\mathbb{R}^{N}}=\left(g^{-1}\left(\mathrm{I}_{\mathrm{d}}, J\right) z\right)^{T}(g q) \\
& =\left\langle z,\left(\mathrm{I}_{\mathrm{d}}, J\right)^{T} q\right\rangle_{\mathbb{R}^{N}}=\langle z, w\rangle_{\mathbb{R}^{N}} .
\end{aligned}
$$

It follows that $v(t)$ moves parallel if

$$
0=P_{x(t)} \dot{v}(t)=\left(\mathrm{I}_{\mathrm{d}}, J(y(t))\right)^{T} g^{-1}(y(t))\left(\mathrm{I}_{\mathrm{d}}, J(y(t))\right) \dot{v}(t), \quad t \in\left[t_{1}, t_{2}\right] .
$$

Definition 5.8. Let $\tilde{v}(t), t \in[0,1]$ be the unique solution to the ODE (10) with boundary conditions $\tilde{v}\left(t_{1}\right)=u\left(t_{1}\right) \in \mathbf{T}_{x\left(t_{1}\right)}$. We say that $u\left(t_{2}\right):=\tilde{v}\left(t_{2}\right) \in \mathbf{T}_{x\left(t_{2}\right)}$ is the parallel transport of the vector $u\left(t_{1}\right)$ along $(x(s), s \in[0,1])$.

For notational clarity the rest of this section uses the Einstein summation convention.

Remark 5.9. Take $v(t)$ and $w(t), t \in\left[t_{1}, t_{2}\right]$, as above and denote $\left(g^{i j}\right)_{i, j=1, \ldots, d}:=g^{-1}$. We say that $w(t)$ is the induced parallel transport along $(y(s), s \in[0,1])$. That is $w(t)$ solves the equation

$$
\begin{aligned}
0 & =g^{-1}(y(t))\left(\mathrm{I}_{\mathrm{d}}, J(y(t))\left[\left(\mathrm{I}_{\mathrm{d}}, J(y(t))^{T} w(t)\right]^{\bullet}\right.\right. \\
& =\dot{w}(t)+g^{-1}(y(t))\left(\mathrm{I}_{\mathrm{d}}, J(y(t))\left[\left(\mathrm{I}_{\mathrm{d}}, J(y(t))\right]^{\bullet} w(t)\right.\right. \\
& =\dot{w}(t)+\left\{\left(g^{i l} \sum_{r} J_{l}^{r} D_{j} J_{k}^{r}\right)(y(t)) \dot{y}^{j}(t) w^{k}(t)\right\}_{i=1, \ldots, d} .
\end{aligned}
$$

Using the notation

$$
\Gamma_{j k}^{i}:=g^{i l} \sum_{r} J_{l}^{r} D_{j} J_{k}^{r}=g^{i l} \sum_{r} J_{l}^{r} D_{j} D_{k} F^{r}, \quad i, j, k \in\{1, \ldots, d\},
$$

the above equation turns into

$$
0=\dot{w}(t)+\left\{\Gamma_{j k}^{i}(y(t)) \dot{y}^{j}(t) w^{k}(t)\right\}_{i=1, \ldots, d} .
$$




\subsection{Riemannian connection}

In this section we use a different definition of $\Gamma$ and instead consider it in the following way.

Definition 5.10. A function

$$
\Gamma=\left(\Gamma_{j k}^{i}(y)\right)_{i, j, k=1, \ldots, d} \quad y \in \mathbb{R}^{d}
$$

$\Gamma \in C^{\infty}\left(\mathbb{R}^{d} \times\{1, \ldots, d\}^{3} \rightarrow \mathbb{R}\right)$ is called a connection i.e. $\Gamma$ is a collection of $d^{3}$ smooth functions on $\mathbb{R}^{d}$. Let $M=\left\{(y, F(y)): y \in \mathbb{R}^{d}\right\}$ be a $C^{\infty}$-manifold of $\mathbb{R}^{N}$ and $g(y):=\left(\mathrm{I}_{\mathrm{d}}, J(y)\right)\left(\mathrm{I}_{\mathrm{d}}, J(y)\right)^{T}, y \in \mathbb{R}^{d}$. The pair $\left(\mathbb{R}^{d}, g\right)$ is called a Riemannian space and the pair $(M, g)$ is called a Riemannian manifold.

Definition 5.11. Let $\Gamma$ be a connection on $\left(\mathbb{R}^{d}, g\right)$ and let $(w(s), s \in[0,1]) \in C^{\infty}([0,1] \rightarrow$ $\left.\mathbb{R}^{d}\right)$ be a vector field along a smooth curve $(y(s), s \in[0,1])$. If equation (12) holds i.e.

$$
0=\dot{w}(t)+\left\{\Gamma_{j k}^{i}(y(t)) \dot{y}^{j}(t) w^{k}(t)\right\}_{i=1, \ldots, d} \quad t \in\left[t_{1}, t_{2}\right]
$$

we say that $w(t)$ is parallel transported along $(y(s), s \in[0,1])$ under $\Gamma$, for $t \in\left[t_{1}, t_{2}\right]$. Remark 5.12. The objects $\Gamma_{j k}^{i}, i, j, k \in\{1, \ldots, d\}$ are called Christoffel symbols.

Definition 5.13. A connection $\Gamma$ on a Riemannian space $\left(\mathbb{R}^{d}, g\right)$ is called Riemannian if the following holds.

(i) For all $i \in\{1, \ldots, d\}$ we have $\Gamma_{j k}^{i}=\Gamma_{k j}^{i}, j, k \in\{1, \ldots, d\}$.

(ii) For vector fields $(w(s), s \in[0,1]),(v(s), s \in[0,1])$ parallel transported along a smooth curve $(y(s), s \in[0,1])$, it holds that

$$
g\left\langle w\left(t_{1}\right), v\left(t_{1}\right)\right\rangle=g\left\langle w\left(t_{2}\right), v\left(t_{2}\right)\right\rangle, \quad t_{1}, t_{2} \in[0,1] .
$$

If the parallel transport under $\Gamma$ preserves inner products in the above sense we say that $\Gamma$ is compatible with $g$.

The following theorem explains why we defined $\Gamma$ by (11).

Theorem 5.14. There is exactly one Riemannian connection on $\left(\mathbb{R}^{d}, g\right)$. This connection is given by

$$
\Gamma_{j k}^{i}=\frac{1}{2} g^{i l}\left(D_{j} g_{l k}+D_{k} g_{l j}-D_{l} g_{j k}\right), \quad i, j, k \in\{1, \ldots, d\} .
$$

Proof: Step 1: (Existence) Let $M=\left\{(y, F(y)): y \in \mathbb{R}^{d}\right\}$ be a $d$-dimensional $C^{\infty}$ manifold in $\mathbb{R}^{N}, J$ the corresponding Jacobian matrix

$$
J=\left(J_{i}^{r}(y)\right)_{i=1, \ldots, d ; r=1, \ldots, n}=\left(\frac{\partial F^{r}}{\partial y^{i}}\right)_{i=1, \ldots, d ; r=1, \ldots, n}
$$


and $g(y)=\left(\mathrm{I}_{\mathrm{d}}, J(y)\right)\left(\mathrm{I}_{\mathrm{d}}, J(y)\right)^{T}, y \in \mathbb{R}^{d}$. Set

$$
\Gamma_{j k}^{i}=g^{i l} \sum_{r} J_{l}^{r} D_{j} D_{k} F^{r}, \quad i, j, k \in\{1, \ldots, d\},
$$

i.e. (11). Under this assumption the symmetry condition (i) is satisfied. Let $(w(s), s \in[0,1])$ and $(v(s), s \in[0,1])$ be vector fields along a smooth curve $(y(s), s \in[0,1])$ in $\mathbb{R}^{d}$, which are parallel transported along this curve under $\Gamma$. Furthermore define

$$
q(s):=\left(\mathrm{I}_{\mathrm{d}}, J(s)\right)^{T} w(s), \quad p(s):=\left(\mathrm{I}_{\mathrm{d}}, J(s)\right)^{T} u(s), \quad s \in[0,1] .
$$

We have

$$
g\langle w(s), v(s)\rangle=q(s)^{T} p(s)
$$

and

$$
(g\langle w(s), v(s)\rangle)^{\bullet}=\dot{q}(s)^{T} p(s)+q(s) \dot{p}(s)^{T}, \quad s \in[0,1] .
$$

Due to (12) and (10), $q(s) \in \mathbf{T}_{x(s)}$ as well as $p(s) \in \mathbf{T}_{x(s)}$ are parallel transported along $x(s)=(y(s), F(y(s)))$ then per definition $\dot{q}(s) \perp \mathbf{T}_{x(s)}$ and $\dot{p}(s) \perp \mathbf{T}_{x(s)}$. It follows that

$$
(g\langle w(s), v(s)\rangle)^{\bullet}=0
$$

i.e. the isometry condition (ii) is satisfied.

Step 2: (Uniqueness) Let $\Gamma$ be a connection and $(y(s), s \in[0,1]),(v(s), s \in[0,1])$ and $(w(s), s \in[0,1])$ be as above. Then from (ii) it holds

$$
\left(w^{i}(s) g_{i j}(s) v^{j}(s)\right)^{\bullet}=0 .
$$

Using (12) i.e. $\dot{w}(s)=-\left\{\Gamma_{j k}^{i}(y(s)) \dot{y}^{j}(s) w^{k}(s)\right\}_{i=1, \ldots, d}$ as well as a similar relation for $\dot{v}(s), s \in[0,1]$ we get

$$
\begin{aligned}
0 & =-\Gamma_{k l}^{i} \dot{y} w^{l} g_{i j} v^{j}+w^{i} \dot{y}^{k} D_{k} g_{i j} v^{j}-w^{i} g_{i j} \Gamma_{k l}^{i} \dot{y}^{k} v^{l} \\
& =\left(-\Gamma_{k l}^{i} g_{i j}+D_{k} g_{l j}-g_{l i} \Gamma_{k j}^{i}\right) w^{l} \dot{y}^{k} v^{j}
\end{aligned}
$$

Thus it holds

$$
\Gamma_{k l}^{i} g_{i j}+\Gamma_{k j}^{i} g_{l i}=D_{k} g_{l j} .
$$

We swap cyclically $k \rightarrow l \rightarrow j \rightarrow k$ to obtain

$$
-\left(\Gamma_{l j}^{i} g_{i k}+\Gamma_{l k}^{i} g_{j i}\right)=-D_{l} g_{j k}
$$

and

$$
\Gamma_{j k}^{i} g_{i l}+\Gamma_{j l}^{i} g_{k i}=D_{j} g_{k l} .
$$

Adding these last three equations together, using the symmetry (i) and the symmetry of $\left(g_{i j}\right)$ we get

$$
2 \Gamma_{j k}^{i} g_{i l}=\left(D_{j} g_{k l}+D_{k} g_{l j}-D_{l} g_{j k}\right)
$$

The statement follows.

Remark 5.15. The matrix $g$ defined by (9) uniquely defines $\Gamma_{j k}^{i}$ in Theorem 5.14. Thus $g$ gives a complete description of the geometry of the smooth Riemannian manifold $(M, g)$. 


\subsection{The orthonormal bundle}

Having defined parallel transport it is natural to define the concept of moving bases.

Definition 5.16. Let $(y(s), s \in[0,1])$ be a smooth curve in $\mathbb{R}^{d}$ and $(x(s), s \in[0,1])$ a smooth curve on $M=\left\{(y, F(y)): y \in \mathbb{R}^{d}\right\}$ with $x(s)=(y(s), F(y(s))), s \in[0,1]$. Let $E=\left\{e_{1}, \ldots, e_{d}\right\}$ be an orthonormal basis (or short ON-basis) in $\mathbf{T}_{x(0)}$ and let $f_{1}, \ldots, f_{d} \in \mathbb{R}^{d}$ where $e_{l}=\left(\mathrm{I}_{\mathrm{d}}, J(y(0))\right)^{T} f_{l}, l=1, \ldots, d$. Also let $\left(f_{l}(s), s \in[0,1]\right)$ be vector fields along $(y(s), s \in[0,1])$ satisfying the differential equation

$$
\left\{\begin{array}{l}
\dot{f}_{s}(s)=\left\{\Gamma_{j k}^{i}(y(s)) \dot{y}^{j}(s) f_{l}^{k}(s)\right\}_{i=1, \ldots, d} \quad s \in[0,1], \quad l=1, \ldots, d . \\
f(0)=f_{l}
\end{array}\right.
$$

We define

$$
e_{l}(s):=\left(\mathrm{I}_{\mathrm{d}}, J(y(s))\right)^{T} f_{l}(s), \quad s \in[0,1], l=1, \ldots, d .
$$

We have that $\left\{e_{1}(s), \ldots, e_{d}(s)\right\}$ is an orthonormal basis in $\mathbf{T}_{x(s)}, s \in[0,1]$ and say that $\left\{e_{1}(s), \ldots, e_{d}(s)\right\}$ is a moving basis along $x(s)$.

These final notions describe how curves behave on a manifold.

Definition 5.17. The family

$$
\left\{\left(x, e_{1}, \ldots, e_{d}\right): x \in M,\left\{e_{1}, \ldots, e_{d}\right\} \text { is an ON-basis on } \mathbf{T}_{x}\right\},
$$

is called the orthonormal bundle and is denoted $O(M)$.

Definition 5.18. We say that a curve $(u(s), s \in[0,1])$ in $O(M)$ is horizontal if $u(s)=$ $(x(s), E(s), s \in[0,1])$, where $E(s)$ is a moving basis along $(x(s), s \in[0,1])$. The curve $(u(s), s \in[0,1])$, is called the horizontal lift of $(x(s), s \in[0,1])$.

Proposition 5.19. Let $(w(s), s \in[0,1])$ be a smooth curve in $\mathbb{R}^{d}$. Then there is a horizontal curve $u(s)$ on $O(M)$ satisfying

$$
\begin{aligned}
& \dot{w}(s)=\left(r^{1}(s), \ldots, r^{d}(s)\right)^{T}, \\
& \dot{x}(s)=E(s) r(s), \\
& x(s)=(y(s), F(y(s))),
\end{aligned}
$$

$s \in[0,1]$. For given initial condition $u_{0}=\left(x_{0}, e_{1}, \ldots, e_{d}\right) \in O(M)$ the horizontal curve $u(s)=((x(s), E(s)), s \in[0,1])$, with $u(0)=u_{0}$ is the unique solution to the system of differential equations

$$
\left\{\begin{array}{l}
\dot{f}_{l}(s)+\left\{\Gamma_{j k}^{i}(y(s)) \dot{y}^{j}(s) f_{l}^{k}(s)\right\}_{i=1, \ldots, d}=0, \quad l=1, \ldots, d, \\
\dot{y}(s)=r^{l}(s) f_{l}(s), \quad s \in[0,1] .
\end{array}\right.
$$

We call the map $w(s) \rightarrow u(s)$ the development map.

Remark 5.20. As a motivation for (15) consider a curve $(w(s), s \in[0,1])$, in $\mathbb{R}^{d}$. Now let $r(s)$ be the velocity along $w(s)$ i.e. $r(s)=\dot{w}(s)$. Solving (15) gives $y(s)$, a curve in $\mathbb{R}^{d}$, and $f_{l}(s), l=1, \ldots, d$, basis vectors in $\mathbb{R}^{d}$. These objects provide an equivalent description of the curve $x(s)$ on $M$, with basis vectors $e_{l}(s), l=1, \ldots, d$, on $\mathbf{T}_{x(s)}$. 


\subsection{Brownian motion on a smooth Riemannian manifold}

In order to construct Brownian motion on smooth Riemannian manifolds we first remind of the following relation between the Itô and Stratonovich integral. Below the multiplications are made in the sense of scalar products in $\mathbb{R}^{m}$.

Definition 5.21. Let $X, Y$ be continuous semimartingales in $\mathbb{R}^{m}$. The Stratonovich integral $\int Y \partial X$ is defined by

$$
S_{t}:=\int_{0}^{t} Y_{s} \partial X_{s}:=\int_{0}^{t} Y_{s} d X_{s}+\frac{1}{2}[Y, X]_{t}
$$

where $t \in T(=[0,1],[0, \infty), \ldots), \int Y d X$ is the usual Itô integral and $[\cdot, \cdot]_{t}$ denotes the covariation. We may write

$$
\partial S=Y \partial X=d S=Y d X+\frac{1}{2} d\langle Y, X\rangle=Y d X+\frac{1}{2} d Y d X
$$

using differential notation.

Remark 5.22. The Stratonovich calculus is compatible with the classical non-stochastic differential calculus. For example for a smooth function, $F \in C^{\infty}\left(\mathbb{R}^{m}\right)$, we have

$$
\begin{aligned}
& \text { (i) } \partial(F(X))=F^{\prime}(X) \partial X, \\
& \text { (ii) } \partial(X Y)=Y \partial X+X \partial Y \text {. }
\end{aligned}
$$

We consider the system of differential equations (15)

$$
\left\{\begin{array}{l}
\dot{y}(s)=f_{m}(s) \dot{W}^{m}(s), \\
\dot{f}_{l}(s)=\left\{-\Gamma_{j k}^{i}(y(s)) f_{m}^{j}(s) f_{l}^{k}(s)\right\}_{i=1, \ldots, d} \dot{W}^{m}(s), \quad l=1, \ldots, d, s \in[0,1],
\end{array}\right.
$$

with initial conditions

$$
\begin{aligned}
& \left(y(0), f_{1}(0), \ldots, f_{d}(0)\right)=\left(y_{0}, f_{1_{0}}, \ldots, f_{d_{0}}\right) \\
& x_{0}:=\left(y, F\left(y_{0}\right)\right), \quad e_{l_{0}}:=\left(\mathrm{I}_{\mathrm{d}}, J\left(y_{0}\right)\right)^{T} f_{l_{0}}, \quad l=1, \ldots, d,
\end{aligned}
$$

such that

$$
\left(x_{0}, e_{1_{0}}, \ldots, e_{d_{0}}\right) \in O(M) .
$$

For $m=1, \ldots, d$ use the notation

$$
\varphi_{m}=\varphi_{m}\left(y(s), f_{1}(s), \ldots, f_{d}(s)\right):=\left\{-\Gamma_{j k}^{i}(y(s)) f_{m}^{j}(s) f_{l}^{k}(s)\right\}_{i, l=1, \ldots, d} .
$$

Note that $\varphi_{m}, m=1, \ldots, d$, is a $d^{2}$-dimensional vector whose entries corresponds to the $d$ different $d$-dimensional parallel transports of the basis vectors $f_{i}, i=1, \ldots, d$. Let

$$
\Phi\left(y(s), f_{1}(s), \ldots, f_{d}(s)\right):=\underbrace{\left[\begin{array}{ccc}
\mid & & \mid \\
f_{1} & \ldots & f_{d} \\
\mid & & \mid \\
-- & -- & -- \\
\mid & & \mid \\
\varphi_{1} & \ldots & \varphi_{d} \\
\mid & & \mid
\end{array}\right]}_{d}
$$


With this notation (15) takes the form

$$
\left(\dot{y}(s), \dot{f}_{1}(s), \ldots, \dot{f}_{d}(s)\right)^{T}=\Phi\left(y(s), f_{1}(s), \ldots, f_{d}(s)\right) \dot{W}(s), s \in[0,1] .
$$

The main idea for constructing Brownian motion on a manifold is to first get a description on how to transfer smooth curves to a manifold via differential calculus. We then wish to replace these smooth curves with the jagged curves of Brownian motion. This is done by replacing the usual differentials with Stratonovich ones. Work has to be done to ensure that this procedure yields a well defined result. We consider the Stratonovich SDE

$$
\left\{\begin{array}{l}
\left(\partial y, \partial f_{1}, \ldots, \partial f_{d}\right)=\Phi\left(y, f_{1}, \ldots, f_{d}\right) \partial \gamma \\
\text { deterministic initial condition }(16)
\end{array}\right.
$$

where $\left(\gamma_{s}, \mathcal{F}_{s}^{\gamma}, s \in[0,1]\right)$ is a $d$-dimensional Brownian motion on a probability space $(\Omega, \mathcal{F}, \mathbb{P})$ equipped with the filtration $\left(\mathcal{F}_{s}\right)_{s \in[0,1]}$. Let

$$
\Psi:=\left(\sum_{q=1}^{i} D_{i} \Phi_{q}^{j}\right)_{i, j=1, \ldots, d(d+1)} .
$$

Since there exist $K>0$ such that for all $\xi, \eta \in \mathbb{R}^{d(d+1)}$

$$
\|\Phi(\xi)-\Phi(\eta)\|+\|\Psi(\xi)-\Psi(\eta)\| \leq K\|\xi-\eta\|
$$

and

$$
\|\Phi(\xi)\|^{2}+\|\Psi(\xi)\|^{2} \leq K\left(1+\|\xi\|^{2}\right)
$$

we may formulate the following theorem.

Theorem 5.23. The Itô SDE

$$
\left\{\begin{array}{l}
d\left(y, f_{1}, \ldots, f_{d}\right)=\Phi\left(y, f_{1}, \ldots, f_{d}\right) d \gamma+\frac{1}{2} \Psi\left(y, f_{1}, \ldots, f_{d}\right) d t \\
(16)
\end{array}\right.
$$

has a unique strong solution. This implies that the Stratonovich SDE (17) has a unique strong solution namely the solution to the Itô SDE (18).

Definition 5.24. (a) Let $\left(\left(\left(y(s), f_{1}(s), \ldots, f_{d}(s)\right), s \in[0,1]\right),\left(\mathcal{F}_{s}\right)_{s \in[0,1]}, \mathbb{P}\right)$ be a solution to (17). Furthermore let

$$
\hat{\gamma}(s):=(y(s), F(y(s))), \quad e_{l}(s):=\left(\mathrm{I}_{\mathrm{d}}, J(y(s))\right)^{T} f_{l}(s), \quad l=1, \ldots, d, s \in[0,1] .
$$

The process $(\hat{\gamma}, E)=\left(\left(\hat{\gamma}(s), e_{1}(s), \ldots, e_{d}(s)\right), s \in[0,1],\left(\mathcal{F}_{s}\right)_{s \in[0,1]}, \mathbb{P}\right)$ is called horizontal Brownian motion.

(b) We call $\hat{\gamma}:=\left((\hat{\gamma}(s), s \in[0,1]),\left(\mathcal{F}_{s}\right)_{s \in[0,1]}, \mathbb{P}\right)$ Brownian motion on $M$.

(c) Let $0 \leq s_{1}, s_{2} \leq 1$ and $v=v^{i} e_{i}\left(s_{1}\right) \in \mathbf{T}_{\hat{\gamma}\left(s_{1}\right)}$. The quantity

$$
T_{s_{1} \rightarrow s_{2}}^{\hat{\gamma}} v:=v^{T} e_{i}\left(s_{2}\right) \text {, }
$$


is called stochastic parallel transport from $\mathbf{T}_{\hat{\gamma}\left(s_{1}\right)}$ to $\mathbf{T}_{\hat{\gamma}\left(s_{2}\right)}$ along $\hat{\gamma}$.

(d) Let $\hat{\gamma}_{0} \in M$. The set of curves $P_{\hat{\gamma}_{0}}(M):=\left\{\hat{\gamma} \in C([0,1] ; M), \hat{\gamma}(0)=\hat{\gamma}_{0}\right\}$ is called the path space over $M$.

(e) The mapping $\hat{\gamma}:=I(\gamma)$

$$
C\left([0,1] ; \mathbb{R}^{d}\right) \ni \gamma(\cdot, \omega) \rightarrow \hat{\gamma}(\cdot, \omega) \in P_{\hat{\gamma}(0)}(M), \mathbb{P} \text {-a.e. } \omega \in \Omega
$$

is called the Itô map.

(f) Let $\nu$ denote the Wiener measure on $\left\{\varphi \in C\left([0,1] ; \mathbb{R}^{d}\right): \varphi(0)=0\right\}$. The image measure $\hat{\nu}:=I_{*} \nu$ under $I$, is called Wiener measure on $P_{\hat{\gamma}_{0}}(M)$.

(g) The mapping $\gamma \rightarrow(\hat{\gamma}, E)$ is called the stochastic development map and the mapping $H: \hat{\gamma} \rightarrow(\hat{\gamma}, E)$ is called stochastic horizontal lift.

The stochastic parallel transport has the following properties.

Proposition 5.25. Let $0 \leq s_{1}, s_{2}, s_{3} \leq 1, v_{1}=v_{1}^{i} e_{i}\left(s_{1}\right) \in \mathbf{T}_{\hat{\gamma}\left(s_{1}\right)}$, and $v_{2}=v_{2}^{i} e_{i}\left(s_{1}\right) \in$ $\mathbf{T}_{\hat{\gamma}\left(s_{1}\right)}$. Then $\mathbb{P}$-a.s.

$$
T_{s_{2} \rightarrow s_{3}}^{\hat{\gamma}} T_{s_{1} \rightarrow s_{2}}^{\hat{\gamma}} v_{1}=T_{s_{1} \rightarrow s_{3}}^{\hat{\gamma}} v_{1}
$$

and

$$
\left\langle v_{1}, v_{2}\right\rangle_{\mathbf{T}_{\hat{\gamma}\left(s_{1}\right)}}=\left\langle T_{s_{1} \rightarrow s_{2}}^{\hat{\gamma}} v_{1}, T_{s_{1} \rightarrow s_{2}}^{\hat{\gamma}} v_{2}\right\rangle_{\mathbf{T}_{\hat{\gamma}\left(s_{2}\right)}}
$$

Proof: Step 1: From Definition 5.24, (19) is clear for fixed $0 \leq s_{1}, s_{2}, s_{3} \leq 1$, $\mathbb{P}$-a.s. The $\mathbb{P}$-a.s. continuity of $e_{1}, \ldots, e_{d}$ implies $\mathbb{P}$-a.s. continuity in the left hand side of (19) in $s_{1}, s_{2}, s_{3}$ as well as for the right hand side of (19) for $s_{1}, s_{3}$. Therefore (19) holds $\mathbb{P}$-a.s. for all $0 \leq s_{1}, s_{2}, s_{3} \leq 1$ and $v_{1} \in \mathbf{T}_{\hat{\gamma}\left(s_{1}\right)}$.

Step 2: Since $e_{1}, \ldots, e_{d}$ is $\mathbb{P}$-a.s. continuous it follows that $v_{1}^{i} e_{i}, v_{2}^{i} e_{i}$ is continuous as well. It is therefore sufficient to prove (20) for fixed $0 \leq s_{1}, s_{2} \leq 1$. We note

$$
\left\langle v_{1}, v_{2}\right\rangle_{\mathbf{T}_{\hat{\gamma}\left(s_{1}\right)}}=\sum_{i, j} v_{1}^{i} v_{2}^{j}\left\langle e_{i}\left(s_{1}\right), e_{j}\left(s_{1}\right)\right\rangle_{\mathbf{T}_{\hat{\gamma}\left(s_{1}\right)}}=\sum_{i} v_{1}^{i} v_{2}^{i}
$$

and

$$
\left\langle T_{s_{1} \rightarrow s_{2}}^{\hat{\gamma}} v_{1}, T_{s_{1} \rightarrow s_{2}}^{\hat{\gamma}} v_{2}\right\rangle_{\mathbf{T}_{\hat{\gamma}\left(s_{2}\right)}}=\left\langle v_{1}^{i} e_{i}\left(s_{2}\right), v_{2}^{j} e_{j}\left(s_{2}\right)\right\rangle_{\mathbf{T}_{\hat{\gamma}\left(s_{2}\right)}}=\sum_{i=1}^{d} v_{1}^{i} v_{2}^{i},
$$

i.e. the left and right hand sides of (20) coincide.

\subsection{Analysis on the path space}

This section contains a brief overview of the calculus on the path space of Brownian motion on a Riemannian manifold. Detailed calculations and more results can be found in [Driver and Röckner, 1992], [Hsu, 1995], [Hsu, 2002]. Let $(\Omega, \mathcal{F}, \mathbb{P})$ be a probability space and $\gamma(\omega)$ be a $d$-dimensional Brownian motion on $(\Omega, \mathcal{F}, \mathbb{P})$ with filtration $\mathcal{F}_{s}$, $s \in[0,1]$ and corresponding measure $\nu$. In addition let $\mathcal{O}(d)$ denote the space of $d \times d$ orthogonal matrices. 
Proposition 5.26. Let $A=\left((A(s), s \in[0,1]),\left(\mathcal{F}_{s}\right)_{s \in[0,1]}, \mathbb{P}\right)$ be an $\mathcal{O}(d)$-valued process and $\alpha=\left((\alpha(s), s \in[0,1]),\left(\mathcal{F}_{s}\right)_{s \in[0,1]}, \mathbb{P}\right)$ be an $\mathbb{R}^{d}$-valued process such that $E\left[\int|\alpha|^{2} d s\right]<\infty$, and $E\left[\int\|A\|^{2} d s\right]<\infty$. Introduce

$$
W(s)=\int_{0}^{s} A(r) d \gamma(r)+\int_{0}^{s} \alpha(r) d r, \quad s \in[0,1] .
$$

We let $\tilde{\nu}:=W_{*} \nu$ denote the image measure under the map $\gamma \mapsto W$. We have

$$
\frac{d \tilde{\nu}}{d \nu}(\gamma)=\exp \left(\int_{0}^{1} \alpha(r) A(r) d \gamma(r)-\frac{1}{2} \int_{0}^{1}|\alpha(r)|^{2} d r\right) .
$$

Proof: The process $\gamma^{\prime}(s)=\int_{0}^{s} A(r) d \gamma(r), s \in[0,1]$ is a Brownian motion with respect to $\left(\mathcal{F}_{s}\right)_{s \in[0,1]}$, since $\gamma^{\prime}$ is a continuous local martingale with quadratic variation

$$
\left[\int_{0} A d \gamma, \int_{0} A d \gamma\right]_{s}=\int_{0}^{s} A^{T} A d[\gamma, \gamma]_{r}=\mathrm{I}_{\mathrm{d}} \cdot s, \quad s \in[0,1] .
$$

That is $\nu\left(\gamma^{\prime}(\omega): \omega \in \Omega\right)=1$ and $W=\gamma^{\prime}+\int \alpha d r$. The statement then follows from the Girsanov formula.

We use the notation

$$
\mathbb{H}:=\left\{h \in C_{0}\left([0,1] ; \mathbb{R}^{d}\right): h \text { absolutely continuous } \int\left|h^{\prime}(s)\right|^{2} d s<\infty\right\}
$$

to denote the $d$-dimensional Cameron-Martin space.

Theorem 5.27. Let $h \in \mathbb{H}$. There exist families $(A(t, \cdot))_{t \in \mathbb{R}},(\alpha(t, \cdot))_{t \in \mathbb{R}}$ of processes such that the following holds.

(i) The conditions of Proposition 5.26 are satisfied for every $t \in \mathbb{R}$.

(ii) It holds that $\alpha(\cdot, s) \in C^{1}\left(\mathbb{R} ; \mathbb{R}^{d}\right), A(\cdot, s) \in C^{1}(\mathbb{R} ; O(d)), s \in[0,1]$, $\mathbb{P}$-a.s. as well as $\mathbb{R}^{d} \ni \alpha(\cdot, 0)=0$ and $O(d) \ni A(\cdot, 0)=0$.

(iii) Let

$$
W(t, s)=\int_{0}^{s} A(t, r) d \gamma(r)+\int_{0}^{s} \alpha(t, r) d r
$$

and define

$$
\xi(t, s):=I(W(t, s)), \quad u(t, s):=H(\xi(t, s)), \quad t \in \mathbb{R}, s \in[0,1],
$$


where I and $H$ are the Itô mapping respectively horizontal lift from Definition 5.24. Then $\xi$ has the representation

$$
\xi(t, s)=\hat{\gamma}(s)+\int_{0}^{t} u(r, s) h(s) d r
$$

and is a solution to the geometric flow equation

$$
\left\{\begin{array}{l}
\frac{d}{d t} \xi(t, s)=u(t, s) h(s)=H(\xi(t, s)) h(s), \\
\xi(0, s)=\hat{\gamma}(s), \quad s \in[0,1], t \in \mathbb{R} .
\end{array}\right.
$$

is satisfied with the solution

Here $\hat{\gamma}$ is a Brownian motion on $M$.

In (21) $u(r, s)$ is to be understood by the mapping $\mathbb{R}^{d} \rightarrow \mathbf{T}_{\xi(r, s)}, u(r, s) k:=k^{i} e_{i}(W(r, s))$ where $e_{i}(W(r, s))$ denotes the walking basis vector $e_{i}$ along $W(r, s)$.

Proof: The proof is omitted but can be found in the papers [Hsu, 1995] and [Hsu, 2002].

We denote

$$
\nu_{t}:=W(t, \cdot)_{*} \mathbb{P}, \quad t \in \mathbb{R},
$$

where $\nu_{0}=\nu$. Furthermore we let $\gamma^{\prime}(t)=\left(\left(\gamma^{\prime}(t, s), s \in[0,1]\right),\left(\mathcal{F}_{s}\right)_{s \in[0,1]}, \mathbb{P}\right)$ given by

$$
\gamma^{\prime}(t, s):=\int_{0}^{s} A(t, r) d \gamma(r), \quad t \in \mathbb{R} .
$$

According to the proof of Proposition $5.26 \gamma^{\prime}(t, \cdot)$ is a Brownian motion with respect to the filtration $\mathcal{F}_{s}, s \in[0,1]$. The map $\gamma(\omega) \rightarrow \gamma^{\prime}(t)(\omega)$ induces an injective map $b: \Omega \rightarrow$ $\Omega$.

The flow equation is used to calculate the directional derivative on the manifold, see (25) below.

Theorem 5.28. (a) (Quasi invariance) The measures $\nu_{t}, t \in \mathbb{R}$ are equivalent and we have for $\mathbb{P}$-a.e. $\omega \in \Omega$

$$
\frac{d \nu_{t}}{d \nu}(\gamma(\omega))=\exp \left\{\int \alpha(t, r) A(t, r) d \gamma(r)-\frac{1}{2} \int|\alpha(t, r)|^{2} d r\right\}(\omega)
$$

(b) Almost surely the Radon-Nikodym derivative $\frac{d \nu_{t}}{d \nu}(\gamma)$ belongs to $C^{1}(\mathbb{R})$ and

$$
\left.\frac{d}{d t}\right|_{t=0} \frac{d \nu_{t}}{d \nu}(\gamma)=\left.\int \frac{d}{d t}\right|_{t=0} \alpha(t, r) d \gamma(r), \quad t \in \mathbb{R} .
$$


Proof: (a) Let

$$
\tilde{\nu}_{t}:=\left(\gamma(s)(\cdot)+\int_{0}^{s} \alpha(t, r)\left(b^{-1} \cdot\right) d r\right)_{*} \nu .
$$

The Girsanov formula yields

$$
\frac{d \tilde{\nu}_{t}}{d \nu}(\gamma(\omega))=\exp \left\{\int_{0}^{1} \alpha(t, r)\left(b^{-1}(\omega)\right) d \gamma(r)(\omega)-\frac{1}{2} \int_{0}^{1}\left|\alpha(t, r)\left(b^{-1}(\omega)\right)\right|^{2} d r\right\} .
$$

On the other hand we have $\nu_{t}=b_{*} \tilde{\nu}_{t}, t \in \mathbb{R}$. Taking into account that $\gamma$ and $\gamma^{\prime}$ are Brownian motions it follows $\nu(d \gamma(\omega))=\nu\left(d \gamma^{\prime}(t)(\omega)\right)$ and

$$
\begin{aligned}
\frac{d \nu_{t}}{d \nu}(\gamma(\omega)) & =\frac{\nu_{t}(d \gamma(\omega))}{\nu(d \gamma(\omega))}=\frac{\tilde{\nu}_{t}\left(d \gamma^{\prime}(t)(\omega)\right)}{\nu(d \gamma(\omega))}=\frac{\tilde{\nu}\left(d \gamma^{\prime}(t)(\omega)\right)}{\nu\left(d \gamma^{\prime}(t)(\omega)\right)}=\frac{d \tilde{\nu}_{t}}{d \nu}(\gamma(b(\omega))) \\
& =\exp \left\{\int_{0}^{1} \alpha(t, r)(\omega) d \gamma^{\prime}(r)(\omega)-\frac{1}{2} \int|\alpha(t, r)(\omega)|^{2} d r\right\} \\
& =\exp \left\{\int_{0}^{1} \alpha(t, r)(\omega) A(t, r)(\omega) d \gamma(\omega)-\frac{1}{2} \int|\alpha(t, r)(\omega)|^{2} d r\right\} .
\end{aligned}
$$

(b) Using the stochastic version of dominated convergence theorem, see [Protter, 2005], and taking under consideration $\alpha(0)=0, A(0)=\mathrm{I}_{\mathrm{d}}$, we obtain (23) by differentiating (22).

Corollary 5.29. (a) (Quasi invariance) The measures $\hat{\nu}_{t}:=I_{*} \nu_{t}, t \in \mathbb{R}$ are equivalent and we have for $\mathbb{P}$-a.e. $\omega \in \Omega$

$$
\frac{d \hat{\nu}_{t}}{d \hat{\nu}}(\hat{\gamma}(\omega))=\frac{d \nu_{t}}{d \nu}(\gamma(\omega))
$$

(b) Almost surely the Radon-Nikodym derivative $\frac{d \hat{\nu}_{t}}{d \hat{\nu}}(\hat{\gamma}) \in C^{1}(\mathbb{R})$ and we have

$$
\left.\frac{d}{d t}\right|_{t=0} \frac{d \hat{\nu}_{t}}{d \hat{\nu}}(\hat{\gamma})=\left.\frac{d}{d t}\right|_{t=0} \frac{d \nu_{t}}{d \nu}(\gamma)
$$

Definition 5.30. A vector field on an open set $N \subset M$ is a smooth map $S: N \rightarrow$ $\mathbf{T}_{N}=\bigcup_{x \in N} \mathbf{T}_{x}$ where $S(x) \in \mathbf{T}_{x}, x \in N$. Let $N_{1}$ be an open subset of $M$ and let $N \subset N_{1}$. If $S$ is a vector field on $N_{1}$ then $\left.S\right|_{N}$ is a vector field on $N$. In particular if $N$ is a smooth curve, $N:=(x(s), s \in[0,1])$ on $M$, we say that $S$ is a vector field along $(x(s), s \in[0,1])$.

Remark 5.31. Let $S$ be a vector field on $N \subset M$. If $x=F(y), y \in \mathbb{R}^{d}$, and $S(x)=$ $(I, J(y)) V(y)$, we can identify $S$ with the first order differential operator $v^{i} \frac{\partial}{\partial y_{i}}$. 


\subsection{Directional derivative, gradient, divergence and integration by parts}

In this section we introduce the geometrical objects corresponding to the usual differential notions. This is done by means of specifying the objects on a class of smooth functions.

Definition 5.32. Let $\hat{\gamma}_{0} \in M$. We define

$$
\begin{aligned}
& \hat{Z}:=\left\{\Phi(\hat{\gamma})=\varphi\left(\hat{\gamma}\left(s_{1}\right), \ldots, \hat{\gamma}\left(s_{k}\right)\right), \hat{\gamma} \in P_{\hat{\gamma}_{0}}(M):\right. \\
&\left.0<s_{1}<\ldots<s_{k}=1, \varphi \in C_{b}^{\infty}\left(M^{k}\right), k \in \mathbb{N}\right\},
\end{aligned}
$$

to be the set of smooth cylindrical functions on $M$.

For $f \in C_{b}^{\infty}(M)$ we define the gradient on the tangent space

$$
\tilde{\nabla} f:=\left(\mathrm{I}_{\mathrm{d}}, J\right)^{T} g^{-1} \nabla f \circ F,
$$

here recall that $F$ denotes the function describing the manifold embedding in $\mathbb{R}^{N}$. With this definition and for $\psi \in C^{1}(\mathbb{R} ; M)$ it follows that

$$
\begin{aligned}
\frac{d}{d t}(f \circ \psi(t)) & =\frac{d}{d t}\left(f \circ F \circ F^{-1} \circ \psi(t)\right)=\left\langle\nabla f \circ F, \frac{d}{d t} F^{-1} \psi(t)\right\rangle_{\mathbb{R}^{d}} \\
& =g\left\langle g^{-1} \nabla f \circ F, \frac{d}{d t} F^{-1} \psi(t)\right\rangle=\left\langle\tilde{\nabla} f(x), \frac{d}{d t} \psi(t)\right\rangle_{\mathbf{T}_{x} M} .
\end{aligned}
$$

Definition 5.33. Let $\Phi \in \hat{Z}$ where $\Phi(\hat{\gamma})=\varphi\left(\hat{\gamma}\left(s_{1}\right), \ldots, \hat{\gamma}\left(s_{k}\right)\right)$. We define

$$
\hat{\mathrm{D}}_{s} \Phi(\hat{\gamma}):=\sum_{i=1}^{k} \chi_{\left[0, s_{i}\right]}(s)\left\{T_{s_{i} \rightarrow s}^{\hat{\gamma}_{i}}\left(\tilde{\nabla}_{i} \varphi\right)\left(\hat{\gamma}\left(s_{1}\right), \ldots, \hat{\gamma}\left(s_{k}\right)\right)\right\}, \quad s \in[0,1],
$$

to be the gradient of $\Phi$. Here $\tilde{\nabla}_{i}$ denotes the operator $\tilde{\nabla}$ corresponding to the $i$ th variable $x\left(s_{i}\right)$.

Remark 5.34. We mention that the difference between $\hat{\mathrm{D}}_{s}$ in Definition 5.33 and Section 6 in Paper I, is a parallel transport from $T_{\gamma(s)}$ to $T_{\gamma(0)}$.

Recall that $\mathbb{H}$ denotes the $d$-dimensional Cameron-Martin space.

Definition 5.35. Let $(\hat{\gamma}, E):=\left(\left(\left(\hat{\gamma}(s), e_{1}(s), \ldots, e_{d}(s)\right), s \in[0,1]\right),\left(\mathcal{F}_{s}\right)_{s \in[0,1]}, \mathbb{P}\right)$ be the process constructed from the solution of the system of equations (15) for fixed initial conditions $\left(\hat{\gamma}(0), e_{1}(0), \ldots, e_{d}(0)\right) \in O(M)$. For $h \in \mathbb{H}$ and $\Phi \in \hat{Z}$ we define the directional derivative in the direction of $h$ as

$$
\hat{D}_{h} \Phi(\hat{\gamma}):=\int_{0}^{1}\left\langle\hat{\mathrm{D}}_{s} \Phi(\hat{\gamma}), \frac{d}{d s} h_{s}^{j} e_{j}(s)\right\rangle_{\mathbf{T}_{\hat{\gamma}(s)}} d s .
$$

Note that $\hat{D}_{h} \Phi(\hat{\gamma})$ is independent of $\left(\hat{\gamma}(0), e_{1}(0), \ldots, e_{d}(0)\right)$. 
We obtain

$$
\begin{aligned}
\hat{D}_{h} \Phi(\hat{\gamma}) & =\int_{0}^{1}\left\langle\hat{\mathrm{D}}_{s} \Phi(\hat{\gamma}), \frac{d}{d s} h_{s}^{j} e_{j}(s)\right\rangle_{\mathbf{T}_{\hat{\gamma}(s)}} d s \\
& =\sum_{i=1}^{k} \int_{0}^{1} \chi_{\left[0, s_{i}\right]}(s) \cdot \frac{d}{d s} h_{s}^{j}\left\langle T_{s_{i} \rightarrow s}^{\hat{\gamma}} \tilde{\nabla}_{i} \varphi, e_{j}(s)\right\rangle_{\mathbf{T}_{\hat{\gamma}(s)}} d s \\
& =\sum_{i=1}^{k} \int_{0}^{1} \chi_{\left[0, s_{i}\right]}(s) \cdot \frac{d}{d s} h_{s}^{j}\left\langle T_{s_{i} \rightarrow 0}^{\hat{\gamma}} \tilde{\nabla}_{i} \varphi, e_{j}(0)\right\rangle_{\mathbf{T}_{\hat{\gamma}_{0}}} d s \\
& =\sum_{i=1}^{k} h_{s_{i}}^{j} \cdot\left\langle T_{s_{i} \rightarrow 0}^{\hat{\gamma}} \tilde{\nabla}_{i} \varphi, e_{j}(0)\right\rangle_{\mathbf{T}_{\hat{\gamma}_{0}}} \\
& =\sum_{i=1}^{k} h_{s_{i}}^{j} \cdot\left\langle\tilde{\nabla}_{i} \varphi, e_{j}\left(s_{i}\right)\right\rangle_{\mathbf{T}_{\hat{\gamma}\left(s_{i}\right)}}
\end{aligned}
$$

as another representation of $\hat{D}_{h} \Phi(\hat{\gamma})$. Finally for the solution $\xi^{h}$ of (21) we have $\mathbb{P}$-a.s.

$$
\begin{aligned}
\left.\frac{d}{d t}\right|_{t=0} \Phi \circ \xi^{h}(t, \cdot) & =\sum_{i=1}^{k}\left\langle\tilde{\nabla} \varphi,\left.\frac{d}{d t}\right|_{t=0} \xi^{h}\left(t, s_{i}\right)\right\rangle_{\mathbf{T}_{\hat{\gamma}\left(s_{i}\right)}} \\
& =\sum_{i=1}^{k}\left\langle\tilde{\nabla} \varphi, u\left(0, s_{i}\right) h\left(s_{i}\right)\right\rangle_{\mathbf{T}_{\hat{\gamma}\left(s_{i}\right)}}=\hat{D}_{h} \Phi(\hat{\gamma}) .
\end{aligned}
$$

Remark 5.36. The limit

$$
\lim _{t \rightarrow 0} \frac{1}{t}\left(\Phi \circ \xi^{h}-\Phi(\hat{\gamma})\right)
$$

exist in $L^{2}=L^{2}\left(P_{\hat{\gamma}_{0}}(M), \hat{\nu}\right)$. Therefore,

$$
\lim _{t \rightarrow 0} \frac{1}{t}\left(\Phi \circ \xi^{h}-\Phi(\hat{\gamma})\right)=\hat{D}_{h} \Phi(\hat{\gamma}) \quad \text { in } L^{2} .
$$

Let $\hat{\nu}$ denote the Wiener measure on $P_{\hat{\gamma}_{0}}(M), \hat{\gamma}_{0} \in M$. We study $\hat{\gamma} \rightarrow \xi^{h}(t)$ trajectorywise as a mapping $C_{\hat{\gamma}_{0}}([0,1] ; M) \rightarrow C_{\hat{\gamma}_{0}}([0,1] ; M)$. For $\Phi$ as before and $\Psi \in \hat{Z}$ such that $\Psi(\hat{\gamma})=\psi\left(\hat{\gamma}\left(s_{1}\right), \ldots, \hat{\gamma}\left(s_{k}\right)\right), \hat{\gamma} \in P_{\hat{\gamma}_{0}}(M), \psi \in C_{b}^{\infty}(M)$ we have

$$
\begin{aligned}
\left\langle\hat{D}_{h} \Phi, \Psi\right\rangle_{L^{2}} & =\left\langle\left.\frac{d}{d t}\right|_{t=0} \Phi \circ \xi^{h}, \Psi\right\rangle_{L^{2}}=\lim _{t \rightarrow 0} \frac{1}{t}\left[\left\langle\Phi \circ \xi^{h}(t), \Psi\right\rangle_{L^{2}}-\langle\Phi, \Psi\rangle_{L^{2}}\right] \\
& =\lim _{t \rightarrow 0} \frac{1}{t}\left[\int \Phi \circ \xi^{h}(t)(\hat{\gamma}) \Psi(\hat{\gamma}) \hat{\nu}(d \hat{\gamma})-\langle\Phi, \Psi\rangle_{L^{2}}\right] \\
& =\lim _{t \rightarrow 0} \frac{1}{t}\left[\int \Phi \Psi \circ \xi^{h}(-t)(\hat{\gamma}) \hat{\nu}\left(d \xi^{h}(-t)(\hat{\gamma})\right)-\langle\Phi, \Psi\rangle_{L^{2}}\right] \\
& =\lim _{t \rightarrow 0} \frac{1}{t}\left[\int \Phi \Psi \circ \xi^{h}(-t)(\hat{\gamma}) \frac{\hat{\nu}\left(d \xi^{h}(-t)(\hat{\gamma})\right)}{\hat{\nu}(d \hat{\gamma})} \hat{\nu}(d \hat{\gamma})-\langle\Phi, \Psi\rangle_{L^{2}}\right] .
\end{aligned}
$$


We use the abbreviations

$$
\frac{d \xi^{h}(-t)_{*} \hat{\nu}}{d \hat{\nu}}=\frac{d \hat{\nu}_{-t}}{d \hat{\nu}} \quad \text { and } \quad z^{h}:=\left.\frac{d}{d t}\right|_{t=0} \frac{d \hat{\nu}_{-t}}{d \hat{\nu}}
$$

From (26) and (27) we obtain the integration by parts formula

$$
\left\langle\hat{D}_{h} \Phi, \Psi\right\rangle_{L^{2}}=\left\langle\Phi,\left.\frac{d}{d t}\right|_{t=0}\left[\Psi \circ \xi^{h}(-t)(\hat{\gamma}) \frac{\hat{\nu}\left(d \xi^{h}(-t)(\hat{\gamma})\right)}{\hat{\nu}(d \hat{\gamma})}\right]\right\rangle_{L^{2}}=\left\langle\Phi,-\hat{D}_{h} \Psi+z^{h} \Psi\right\rangle_{L^{2}} .
$$

Let $g \in L^{2}\left([0,1] \rightarrow \mathbb{R}^{d}\right)$ and $h:=\int_{0}^{\bullet} g_{s} d s$. We note that $h \in \mathbb{H}$. Furthermore let $\Phi$ and $\Psi$ be cylindrical functions. We have

$$
\begin{gathered}
\iint_{0}^{1}\left\langle D_{s} \Phi, \Psi g^{j} e_{j}(s)\right\rangle_{\mathbf{T}_{\cdot(s)}} d s d \hat{\nu}=\iint_{0}^{1}\left\langle D_{s} \Phi, g^{j} e_{j}(s)\right\rangle_{\mathbf{T}_{\cdot(s)}} d s \Psi d \hat{\nu}=\left\langle\hat{D}_{h} \Phi, \Psi\right\rangle_{L^{2}} \\
\quad=\left\langle\Phi,-\hat{D}_{h} \Psi+z^{h} \Psi\right\rangle_{L^{2}}=\left\langle\Phi,-\sum_{i=1}^{k} h^{j}\left(s_{i}\right)\left\langle\tilde{\nabla}_{i} \psi, e_{j}\left(s_{i}\right)\right\rangle_{\mathbf{T}_{\cdot\left(s_{i}\right)}}+z^{h} \Psi\right\rangle_{L^{2}}
\end{gathered}
$$

where for the second last equality we have used the above integration by parts formula.

Definition 5.37. We define

$$
\hat{\delta}\left(\Psi g^{j} e_{j}\right):=-\sum_{i=1}^{k} \sum_{j=1}^{d} h^{j}\left(s_{i}\right)\left\langle\tilde{\nabla}_{i} \psi, e_{j}\left(s_{i}\right)\right\rangle_{\mathbf{T}_{\cdot_{\left(s_{i}\right)}}}+z^{h} \Psi
$$

and call it the divergence of $\Psi g^{j} e_{j}$.

Remark 5.38. The divergence is the adjoint operator to the gradient. This is true also for the non-geometric Malliavin gradient and divergence. That is one of the most important relations in Malliavin calculus.

\subsection{Tensors and Ricci curvature}

This presentation of the theory is based on the book [Kühnel, 2002].

Definition 5.39. An $(r, s)$-tensor, $A$, at a point $x \in M$, is a multilinear map

$$
\underbrace{\left(\mathbf{T}_{x}\right)^{*} \times \ldots \times\left(\mathbf{T}_{x}\right)^{*}}_{r} \times \underbrace{\left(\mathbf{T}_{x}\right) \times \ldots \times\left(\mathbf{T}_{x}\right)}_{s} \rightarrow \mathbb{R} .
$$

Here $\left(\mathbf{T}_{x}\right)^{*}$ denotes the dual space to the tangent space at $x$.

Let us a consider a vector field $S$ on $M$. Let $x=F(y), y=\left(y_{1}, \ldots, y_{d}\right) \in \mathbb{R}^{d}$, and $S(x)=(I ; J(y)) V(y)$. We can then identify $S$ with the first order differential operator

$$
S(x)=\left.\sum_{i=1}^{d} v^{i}(y) \frac{\partial}{\partial y_{i}}\right|_{x}
$$


Using the Christoffel symbols of (11) we may introduce the Riemann curvature tensor $R$ in coordinate form as the object

$$
R_{i j k}^{s}:=\frac{\partial \Gamma_{i k}^{s}}{\partial x_{j}}-\frac{\partial \Gamma_{i j}^{s}}{\partial x_{k}}+\Gamma_{i k}^{r} \Gamma_{r j}^{s}-\Gamma_{i j}^{r} \Gamma_{r k}^{s} .
$$

We define

$$
R\left(\frac{\partial}{\partial x_{j}}, \frac{\partial}{\partial x_{k}}\right) \frac{\partial}{\partial x_{i}}=\sum_{s=1}^{d} R_{i j k}^{s} \frac{\partial}{\partial x_{s}} .
$$

Thus for vector fields, $X=\left(\xi_{1}, \ldots, \xi_{d}\right), Y=\left(\eta_{1}, \ldots, \eta_{d}\right)$, and $Z=\left(\zeta_{1}, \ldots, \zeta_{d}\right)$ on $M$, we get the curvature tensor

$$
\begin{aligned}
R(X, Y) Z & =R\left(\sum_{i=1}^{d} \xi_{i} \frac{\partial}{\partial y_{i}}, \sum_{j=1}^{d} \eta_{j} \frac{\partial}{\partial y_{j}}\right) \sum_{k=1}^{d} \zeta_{k} \frac{\partial}{\partial y_{k}} \\
& =\sum_{i, j, k=1}^{d} \xi_{i} \eta_{j} \zeta_{k} R\left(\frac{\partial}{\partial y_{i}}, \frac{\partial}{\partial y_{j}}\right) \frac{\partial}{\partial y_{k}} \\
& =\sum_{i, j, k, s=1}^{d} \xi_{i} \eta_{j} \zeta_{k} R_{k i j}^{s} \frac{\partial}{\partial x_{s}} .
\end{aligned}
$$

Note that the Riemann curvature tensor is a $(1,3)$-tensor. Using the Riemann curvature tensor we now define the Ricci tensor.

Definition 5.40. The Ricci tensor is a $(0,2)$-tensor given by the trace of the curvature tensor. That is for an ON-basis $\left(E_{1}, \ldots, E_{d}\right)$

$$
\operatorname{Ric}(Y, Z)(x):=\operatorname{Ric}(X \mapsto R(X, Y) Z)=\sum_{i=1}^{d}\left\langle R\left(E_{i}(x), Y(x)\right) Z(x), E_{i}(x)\right\rangle_{\mathbf{T}_{x}}
$$

Remark 5.41. We recall that $\frac{\partial}{\partial y_{1}}, \ldots, \frac{\partial}{\partial y_{d}}$ is a basis for $\mathbf{T}_{x}$ at $x$. In the same way $d y_{1}, \ldots, d y_{d}$ is a basis for the dual space $\left(\mathbf{T}_{x}\right)^{*}$ with

$$
\left.d y_{i}\right|_{x}\left(\left.\frac{\partial}{\partial y_{j}}\right|_{x}\right)=\delta_{i j}= \begin{cases}1, & i=j \\ 0, & i \neq j\end{cases}
$$

In the same way

$$
\left(\left.\left.d y_{i}\right|_{x} \otimes d y_{j}\right|_{x}\right)\left(\left.\left.\frac{\partial}{\partial y_{k}}\right|_{x} \otimes \frac{\partial}{\partial y_{l}}\right|_{x}\right)=\delta_{i k} \delta_{j l}= \begin{cases}1, & i=k, j=l \\ 0, & \text { otherwise }\end{cases}
$$

Remark 5.42. Taking into account (29) and Remark 5.41 we note that the Ricci tensor has representation

$$
\operatorname{Ric}=R_{i j} d y_{i} \otimes d y_{j}
$$


It follows that the Ricci tensor is given by the matrix described by

$$
R_{j k}=\sum_{i=1}^{d} R_{i j k}^{i} .
$$

This gives an intuitive access to Ricci curvature in the form of a matrix. 



\section{References}

[Di Girolami et al., 2014] Di Girolami, C., Fabbri, G., and Russo, F. (2014). The covariation for banach space valued processes and applications. Metrika, 77(1):51-104.

[Di Girolami and Russo, 2014] Di Girolami, C. and Russo, F. (2014). Generalized covariation for banach space valued processes, itô formula and applications. Osaka $J$. Math., 51(3):729-783.

[Driver and Röckner, 1992] Driver, B. and Röckner, M. (1992). Construction of diffusions on path and loop spaces of compact riemannian manifolds. C. R. Acad. Sci. Paris, 315(5):603-608.

[Fukushima et al., 2011] Fukushima, M., Oshima, Y., and Takeda, M. (2011). Dirichlet Forms and Symmetric Markov Processes. De Gruyter.

[Hsu, 1995] Hsu, E. P. (1995). Quasi-invariance of the wiener measure on the path space over a compact riemannian manifold. J. Funct. Anal., 134(2):417-450.

[Hsu, 2002] Hsu, E. P. (2002). Quasi-invariance of the wiener measure on path spaces: noncompact case. J. Funct. Anal., 193(2):278-290.

[Karlsson, 2013] Karlsson, J. (2013). A class of infinite dimensional stochastic processes with unbounded diffusion. Licentiate thesis, Linköping University.

[Kühnel, 2002] Kühnel, W. (2002). Differential geometry. Curves-surfaces-manifolds, volume 16 of Student Mathematical Library. American Mathematical Society, Providence, RI.

[Löbus, 1995] Löbus, J.-U. (1995). Stochastic differential geometry. Lecture notes, Friedrich-Schiller Universität, Jena.

[Löbus, 2004] Löbus, J.-U. (2004). A class of processes on the path space over a compact riemannian manifold with unbounded diffusion. Trans. Amer. Math. Soc., 356(9):3751-3767.

[Ma and Röckner, 1992] Ma, Z.-M. and Röckner, M. (1992). Introduction to the Theory of (Non-symmetric) Dirichlet Forms. Springer, Berlin.

[Meyer, 2009] Meyer, P.-A. (2009). Stochastic processes from 1950 to the present. Electronic Journ@1 for History of Probability and Statistics, 5(1). 
[Nualart, 2006] Nualart, D. (2006). The Malliavin Calculus and related topics. Springer.

[Nualart, 2009] Nualart, D. (2009). The Malliavin Calculus and Its Applications. AMS, Providence, RI.

[Protter, 2005] Protter, P. E. (2005). Stochastic Integration and Differential Equations. Springer, Berlin.

[Rogers and Williams, 2000] Rogers, L. C. G. and Williams, D. (2000). Diffusions, Markov Processes and Martingales, volume 2. Cambridge University Press, Cambridge.

[Russo and Vallois, 2007] Russo, F. and Vallois, P. (2007). Elements of stochastic calculus via regularization. Lecture Notes in Math., 1899:147-185.

[Wang and Wu, 2008] Wang, F.-Y. and Wu, B. (2008). Quasi-regular dirichlet forms on riemannian path and loop spaces. Forum Math. Volume, 20(6):1085-1096.

[Wang and Wu, 2009] Wang, F.-Y. and Wu, B. (2009). Quasi-regular dirichlet forms on free riemannian path spaces. Infin. Dimens. Anal. Quantum Probab. Relat. Top., 12(2):251-267. 


\section{Papers}

The articles associated with this thesis have been removed for copyright reasons. For more details about these see:

http://urn.kb.se/resolve?urn=urn:nbn:se:liu:diva-121636 